\title{
Fibulin-4 promotes osteosarcoma invasion and metastasis by inducing epithelial to mesenchymal transition via the PI3K/Akt/mTOR pathway
}

\author{
DONG ZHANG ${ }^{1}$, SONGGANG WANG ${ }^{1}$, JIE CHEN $^{2}$, HAITAO LIU ${ }^{3}$, JINFA LU $^{3}$, \\ HUA JIANG $^{3}$, AIMIN HUANG ${ }^{3}$ and YUNZHEN CHEN ${ }^{1}$ \\ ${ }^{1}$ Department of Orthopedics, Qilu Hospital, Shandong University; ${ }^{2}$ Department of Maternal and Child Health, \\ School of Public Health, Shandong University, Jinan, Shandong 250012; ${ }^{3}$ Department of Orthopedics, \\ Xiangcheng No. 2 People's Hospital, Suzhou, Jiangsu 215143, P.R. China
}

Received December 7, 2016; Accepted March 13, 2017

DOI: 10.3892/ijo.2017.3921

\begin{abstract}
This study explored the role of fibulin-4 in osteosarcoma progression and the possible signaling pathway involved. Fibulin-4 mRNA and protein expression in normal tissue, benign fibrous dysplasia, osteosarcoma, osteosarcoma cell lines, the normal osteoblastic cell line hFOB, and different invasive subclones were evaluated by immunohistochemistry (IHC) or immunocytochemistry (ICC) and real-time reverse transcriptase-polymerase chain reaction (real-time qRT-PCR). Using in vitro functional assays, we analyzed the invasive and proliferative abilities of different osteosarcoma cell lines and subclones with differing invasive potential. To assess the role of fibulin-4 in the invasion and metastasis of osteosarcoma cells, lentiviral vectors with fibulin-4 small hairpin RNA (shRNA) and pLVX-fibulin- 4 were constructed and used to infect the highly invasive and low invasive subclones and osteosarcoma cell lines. The effects of fibulin- 4 knockdown and upregulation on the biological behavior of osteosarcoma cells were investigated by functional in vitro and in vivo assays. The results revealed that fibulin-4 expression was upregulated in osteosarcoma, and was positively correlated with low differentiation, lymph node metastasis, and poor prognosis. Fibulin-4 was also found to be over-expressed in highly invasive cell lines and in the highly invasive subclones. Fibulin- 4 could promote osteosarcoma cell invasion and metastasis by inducing EMT via the PI3K/AKT/mTOR pathway. Collectively, our findings demonstrate that fibulin- 4 is a promoter of osteosarcoma development and progression, and suggest a novel therapeutic target for future studies.
\end{abstract}

Correspondence to: Dr Yunzhen Chen, Department of Orthopedics, Qilu Hospital, Shandong University, Jinan, Shandong 250012, P.R. China

E-mail: yunzhenchen888@126.com

Key words: fibulin-4, osteosarcoma, PI3K/AKT/mTOR signaling pathway, epithelial-mesenchymal transition, invasion, metastasis

\section{Introduction}

Osteosarcoma is a malignant tumor originating from mesenchymal tissues. It commonly arises in the distal femur and proximal tibia; it is highly malignant and has poor prognosis. Its clinical characteristics include early pulmonary metastasis, high disability rate, and high recurrence rate. Osteosarcoma treatment in 1970 was limited to amputation and radiotherapy, and most patients died of lung metastasis within two years with a 5-year survival rate of only $10-20 \%(1,2)$. In the last 30 years, the wide application of combinatorial chemotherapy and active surgical resection has greatly improved the 5-year survival rate for osteosarcoma. However, though chemotherapy plays a pivotal role in osteosarcoma treatment, its overall efficacy is still approximately $60 \%$. Thus, osteosarcoma therapy is now in a phase, where it is necessary to explore osteosarcoma markers to determine its biological characteristics and prognosis, and to determine novel clinical therapeutic targets for effective molecular therapy.

Fibulin-4 (epidermal growth factor-containing fibulinlike extracellular matrix protein 2, also known as EFEMP2, MBP1, UPH1) is a member of the fibulin family of glycoproteins. Fibulins, encoded by the FBLN genes, are extracellular matrix proteins. Presently, the fibulin family contains seven members: fibulin-1, $-2,-3,-4,-5,-6$, and $-7(3,4)$. It is mainly involved in the formation and stability of membranes, elastic fibers, and loose connective tissues. Fibulin- 4 is widely distributed in human tissues, and is closely associated with the basement membrane and the extracellular matrix of elastic fibers. Fibulin- 4 plays an important role in the stabilization of extracellular matrix structure $(5,6)$.

Fibulin family members are involved in the processes of cell morphology maintenance, growth, adhesion, and movement, and are closely associated with the development of multiple tumors (6). Fibulin-1 expression is enhanced in ovarian cancer (7) and breast cancer (8) and promotes tumor development. However, in hepatocellular carcinoma (9), gastric cancer (10), and prostate cancer (11), fibulin-1 could suppress tumor growth, induce apoptosis, and inhibit tumor angiogenesis (12). Fibulin-2 as a tumor suppressor gene, could inhibit 
tumor cell growth and invasion in HCC and breast cancer, and inhibited angiogenesis $(13,14)$. Fibulin-3 is reported to be highly expressed in pancreatic cancer $(15)$, cervical cancer $(16,17)$, and glioma (18), and promotes tumor development. However, in nasopharyngeal carcinoma (19), breast cancer (20), and glioblastoma (21), fibulin-3 is downregulated, and can inhibit tumor cell proliferation, invasion, and metastasis. Fibulin-5 is widely considered a tumor suppressor gene that inhibits tumor growth, invasion, and angiogenesis in the development of most tumors (22-25).

At present, studies on the relationship between tumors and fibulin- 4 are still in the initial stage, and few related studies are available. In our current study, we investigated the function of fibulin-4 in human osteosarcoma invasion and metastasis, and the the relationship between fibulin-4 and EMT.

\section{Materials and methods}

Cell culture. Osteosarcoma cell lines (HOS, MG63 and $\mathrm{U}-2 \mathrm{OS}$ ) and the normal osteoblastic cell line hFOB, were obtained from the Shanghai Institute for Biological Sciences, Chinese Academy of Sciences. All cell lines were cultured in complete growth media containing DMEM/F12 (Gibco BRL, Rockville, MD, USA) supplemented with $10 \%$ FBS (Gibco BRL) and $1 \%$ antibiotics, and were maintained at $37^{\circ} \mathrm{C}$ in an incubator with $5 \% \mathrm{CO}_{2}$.

Isolation of MG63 cell subclones. MG63 cells at the logarithmic growth phase were collected and diluted to approximately $10 \mathrm{cells} / \mathrm{ml}$, and were then seeded into a 96-well plate with $0.1 \mathrm{ml} /$ well. Thus, as far as possible, there was only one cell in each well. After 1 week at $37^{\circ} \mathrm{C}$ with $5 \% \mathrm{CO}_{2}$, a single clone from one well was selected and cultured as a subclone. Then the cell electrophoretic mobility (EPM) of each clone was measured to study charge property using microcapillary electrophoresis (microCE) chips according to the method of Omasu et al (26). Moreover, the invasive and proliferative abilities of the highly invasive and low invasive subclones were analyzed by in vitro and in vivo functional assays $(27,28)$. All data are expressed as mean \pm standard error (SE).

Osteosarcoma tissue samples. With informed consent from patients, 290 specimens were obtained from the Department of Pathology, Shandong Qilu Hospital. None of these patients had undergone preoperative radiation or chemotherapy. All patients received regular follow-up. During the study period, contact with 15 patients was lost and 36 patients died. The follow-up period was from 2005 to 2014. This study was approved by the Institutional Medical Ethics Committee of Shandong University.

Immunohistochemistry (IHC) and immunocytochemistry (ICC). For IHC, paraffin-embedded sections were de-waxed in xylene and rehydrated in ethanol. Heat-induced epitope retrieval was then performed using a pressure cooker in $0.01 \mathrm{M}$ citrate buffer at $\mathrm{pH}$ 6.0. The sections were incubated for $3 \mathrm{~min}$ after the cooker reached full pressure. For ICC, cells at $75-80 \%$ confluency were seeded into a cell culture dish containing coverslips. After $24 \mathrm{~h}$, the coverslips were harvested, washed thrice with PBS, and fixed in $95 \%$ ethanol for $30 \mathrm{~min}$.
According to the procedure of the streptavidin-peroxidase detection kit (ZSGB-BIO, Beijing, China), the following steps were common for both IHC and ICC. All sections and coverslips were treated with $3 \%$ hydrogen peroxide $\left(\mathrm{H}_{2} \mathrm{O}_{2}\right)$ and goat serum for $30 \mathrm{~min}$ sequentially, to block endogenous peroxidase and the non-specific binding sites, and then incubated with rabbit anti-human fibulin-4, alkaline phosphatase (ALP), and cysteine-rich angiogenic inducer 61 (CYR61) antibodies (ab125073, ab108337, ab24448; Abcam) at working dilutions of 1:200 overnight at $4^{\circ} \mathrm{C}$. The sections and coverslips were then incubated with the anti-rabbit biotin-conjugated secondary antibody for $30 \mathrm{~min}$ at room temperature, stained for $1-5 \mathrm{~min}$ with the enzyme substrate 3', 3-diaminobenzidine tetrahydrochloride (DAB, Sigma-Aldrich, St. Louis, MO, USA), and counterstained for $5 \mathrm{~min}$ with hematoxylin. Paraffin-embedded sections of human ovarian cancer specimens (fibulin-4-positive) were used as positive controls (29), and the negative control was obtained by replacing the primary antibody with PBS. Brown granules in the cytoplasm or stroma were considered as positive fibulin-4 expression.

Immunohistochemistry (IHC) and immunocytochemistry (ICC) analysis. To assess fibulin-4 expression in IHC and ICC experiments, the stained cell percentage and staining intensity were measured. The percentage of positively stained cells was scored from 0 to 4 (score $0,0 \%$ cells stained; score $1,1-25 \%$; score 2, 26-50\%; score 3,51-75\%; or score $4,76-100 \%$ ), whereas the staining intensity of fibulin- 4 was scored as 0 (negative), 1 (weak), 2 (moderate), or 3 (strong) (30). Taken together, the intensity and percentage scores made up of the final staining score (0-7), and the scores of $0,1-3,4-5$, and 6-7 were converted into sum indices,,-+++ , and +++ , respectively. For statistical analysis, low fibulin-4 expression was defined as - or + , whereas high fibulin- 4 expression was indicated by ++ or +++ . Each tissue section was independently analyzed by three pathologists. Immunohistochemical expression was evaluated using Image-Pro Plus 6.0 (Media Cybernetics, Inc., Rockville, MD, USA) to detect photodensity. In brief, 5 positive fields within a section were selected at random and read using Image-Pro Plus 6.0. The mean densities were subsequently calculated. Using Pearson's product-moment correlation coefficient, the associations between fibulin-4 vs. ALP and CYR61 were analyzed.

Lentivirus transfection. The pLVX-fibulin-4 vector and fibulin-4 shRNA as well as a negative control were obtained from GeneChem Inc. (Shanghai, China). According to the manufacturer's instructions, prior to viral infection, target cells were plated at $0.5 \times 10^{5}$ cells per well in a 24 -well plate and incubated at $37^{\circ} \mathrm{C}$ in a $\mathrm{CO}_{2}$ incubator for 24 or $48 \mathrm{~h}$ until the cells were $60-80 \%$ confluent. The cells were then infected by adding the viral stock at a multiplicity of infection (MOI) of 100. In addition, a transduction well with negative control viral constructs was included. The cells were incubated overnight at $37^{\circ} \mathrm{C}$ with $5 \% \mathrm{CO}_{2}$; the transfection mixture was then replaced with normal complete growth medium to avoid cell toxicity. At the end of $48 \mathrm{~h}$ of incubation, the cells were assessed by fluorescence microscopy. The transfection efficiency was confirmed by western blotting, real-time quantitative RT-PCR, and immunocytochemistry (ICC). The siRNA 
sequence for fibulin-4 was: sense 5'-CAGAUCCGUGCUGG AAACUCG-3' and antisense 3'-AGUUUCCAGCACGGAU CUGAA-5'. The negative control (scrambled order) was: sense 5'-AUCGACGAUCCCUAUUGGCGU-3' and antisense 3'-GCCAAUAGGGAUCGUCGAUCU-5'.

Quantitative real-time-polymerase chain reaction ( $q R T-P C R$ ). TRIzol $^{\circledR}$ Reagent (Ambion ${ }^{\mathrm{TM}}$ ) was used to isolate total RNA from cell and tissue samples. Reverse Transcription was carried out with TaqMan ${ }^{\circledR}$ Reverse Transcription Reagents (Applied Biosystems Inc.; Thermo Fisher Scientific, Inc.). The procedure was based on the protocol provided by Invitrogen. The real-time PCR mixture volume was $25 \mu \mathrm{l}$ including $12.5 \mu \mathrm{l} \mathrm{SYBR}$ Green Mix (Power SYBR ${ }^{\circledR}$ Green PCR Master Mix, Applied Biosystems Inc.), $0.2 \mu \mathrm{l}$ cDNA, $1 \mu 1$ primer pair $\operatorname{mix}(5 \mathrm{pmol} / \mu \mathrm{l}$ each primer), and $11.3 \mu \mathrm{l}$ DNAse/RNAse-free $\mathrm{H}_{2} \mathrm{O}$. The experiment was then set up with the following PCR program on ABI Prism SDS 7000 (Applied Biosystems Inc.; Thermo Fisher Scientific, Inc.): $50^{\circ} \mathrm{C}$ for $2 \mathrm{~min}, 1$ cycle; $95^{\circ} \mathrm{C}$ for $10 \mathrm{~min}, 1$ cycle; 40 cycles of $95^{\circ} \mathrm{C}$ for $15 \mathrm{sec} \rightarrow 60^{\circ} \mathrm{C}$ for $30 \mathrm{sec} \rightarrow 72^{\circ} \mathrm{C}$ for $30 \mathrm{sec} ; 72^{\circ} \mathrm{C} 10 \mathrm{~min}, 1$ cycle. Finally, the results were analyzed with SDS 7000 software. Specific primers were designed by LightCycler ${ }^{\circledR}$ Probe Design software (Roche Diagnostics, Basel, Switzerland) and were synthesized by Takara Biotechnology Co., Ltd. The primer sequences were as follows: fibulin-4: 5'-GCTGCTACTGTTGCTCTTGGG-3', 5'-GGGATGGTCAGACACTCGTTG-3'; E-cadherin: 5'-GGA TTGCAAATTCCTGCCATTC-3', 5'-AACGTTGTCCCG GGTGTCA-3'; N-cadherin: 5'-GTAGCTAATCTAACTG TGACCGATAAGG-3', 5'-TTGGTTTGACCACGGTGAC TAA-3'; vimentin: 5'-GCAGGAGGCAGAAGAATGGTA-3', 5'-GGGACTCATTGGTTCCTTTAAGG-3'; Snail: 5'-TCG GAAGCCTAACTACAGCGA-3', 5'-AGATGAGCATTGGC AGCGAG-3'; Slug: 5'-TGTGACAAGGAATATGTGAGCC-3', 5'-TGAGCCCTCAGATTTGACCTG-3'; Twist: 5'-AGCAA GATTCAGACCCTCAAGCT-3', 5'-CCTGGTAGAGGAAG TCGATGTACCT-3'; $\beta$-actin: 5'-CCACGAAACTACCTT CAACTCCA-3', 5'-GTGATCTCCTTCTGCATCCTGTC-3'.

Western blotting. Cells were lysed on ice in RIPA (radioimmunoprecipitation assay) buffer with $1 \mathrm{mM}$ PMSF (phenylmethylsulfonyl fluoride). From the cell lysate, $40 \mu \mathrm{g}$ of total protein was loaded into the wells of the SDS-PAGE (sodium dodecyl sulfate polyacrylamide gel electrophoresis) gel, along with a molecular weight marker. Electrophoresis was carried out for 1-2 $\mathrm{h}$ at $100 \mathrm{~V}$, followed by transfer to PVDF (polyvinyl difluoride) membranes, which were then blocked with 5\% BSA (bovine serum albumin). The membranes were then incubated overnight at $4^{\circ} \mathrm{C}$ with primary antibodies (E-cadherin sc-8426, N-cadherin sc-7939, vimentin sc-6260, Santa Cruz; fibulin-4 ab125073, Snail ab167609, Slug ab27568, Twist ab50887, PI3K ab86714, p-PI3K ab182651, AKT ab8805, P-AKT 38449, mTOR ab32028, p-mTOR ab109268; Abcam) at working dilutions of 1:1000. After washing the membranes thrice with TBST for 5 min each, the membranes were incubated with conjugated secondary antibody diluted to 1:1000, at room temperature for $1 \mathrm{~h}$. Blots were developed using the enhanced chemiluminescence method (Pierce ${ }^{\mathrm{TM}}$ ECL Western Blotting Substrate; Thermo Fisher Scientific, Inc.).
Growth curves. Cells at the logarithmic phase were collected, seeded into the wells of a 24 -well plate $\left(1 \times 10^{4}\right.$ cells/well) and cultured at $37^{\circ} \mathrm{C}$ with $5 \% \mathrm{CO}_{2}$. Three wells were harvested every day and the cells were counted and averaged. Growth curves were then plotted according to the average cell counts of 7 consecutive days.

Soft agar colony formation assay. DMEM with 20\% FBS $(1.5 \mathrm{ml})$ mixed with $1.5 \mathrm{ml}$ of $1.2 \%$ agar was added to $3.5 \mathrm{~cm}$ dishes and solidified for the bottom layer. Next, $1.5 \mathrm{ml}$ of $0.7 \%$ agar was mixed with $1.5 \mathrm{ml}$ DMEM (20\% FBS) and $200 \mu \mathrm{l}$ of cell suspension (containing 600 cells), and was immediately added to the above culture dishes. All the dishes were incubated for 2 weeks at $37^{\circ} \mathrm{C}$ with $5 \% \mathrm{CO}_{2}$. The assay was repeated in triplicate. Under an inverted microscope (Nikon Eclipse), the dish was divided into quadrants and in each quadrant, colonies with diameters of more than $2 \mathrm{~mm}$ were counted and the average was calculated. All the data are expressed as mean $\pm \mathrm{SE}$.

Cell invasion assay and migration assay. The in vitro Matrigel invasion assay was performed as previously described (31). The polyvinylpyrrolidone-free polycarbonate (PVPF) membrane of Boyden chambers (BD Biosciences, Bedford, MA, USA) were coated with $50 \mu \mathrm{l}$ of Matrigel 1:3 diluted with serum-free media. Cell suspensions in volumes of $200 \mu 1$ ( $2 \times 10^{5}$ cells) were seeded into the upper chambers, and $600 \mu 1$ of serumfree culture supernatant of NIH3T3 cells was added to the lower chamber as a chemotactic factor. The Boyden chambers were then incubated at $37^{\circ} \mathrm{C}$ for $24 \mathrm{~h}$. The non-invading cells on the upper surface of the membrane were removed, and the cells on the lower surface were fixed with $4 \%$ paraformaldehyde, stained with hematoxylin and eosin (H\&E), and counted in five random high-power fields (HPF) under an inverted microscope. The cell migration assay was simultaneously performed with the above steps, without the Matrigel coating on the membrane and an incubation time of only $12 \mathrm{~h}$. The cell invasion and migration assays were both repeated in triplicate. All data are expressed as mean $\pm \mathrm{SE}$.

Tumor xenografts in nude mice. BALB/C-nu/nu nude mice were purchased from the National Resource Center for Rodent Laboratory Animal of China. Each group included 5 nude mice, each of which was inoculated subcutaneously with $5.0 \times 10^{6}$ cells. The mice were maintained in a sterile animal facility and monitored daily for tumor growth. Every week, the tumor volumes were measured using vernier calipers, and calculated according to the formula, $\mathrm{V}=$ length $\mathrm{x}$ width ${ }^{2} \mathrm{x} 0.25$. After 2 months, the mice were sacrificed and the tumors were dissected and examined histologically. All data are expressed as mean \pm SE. The animal experiment was approved by the Institutional Animal Care and Use Committee of Shandong University and was in compliance with all regulatory guidelines.

Statistical analysis. IHC data were analyzed using a $\chi^{2}$ test. A two-tailed t-test was used to compare the means between two sets, and a one-way analysis of variance was used to compare the means among three groups. By the Kaplan-Meier method and the log-rank test, survival curve analysis was performed 


\section{A Fibulin-4}
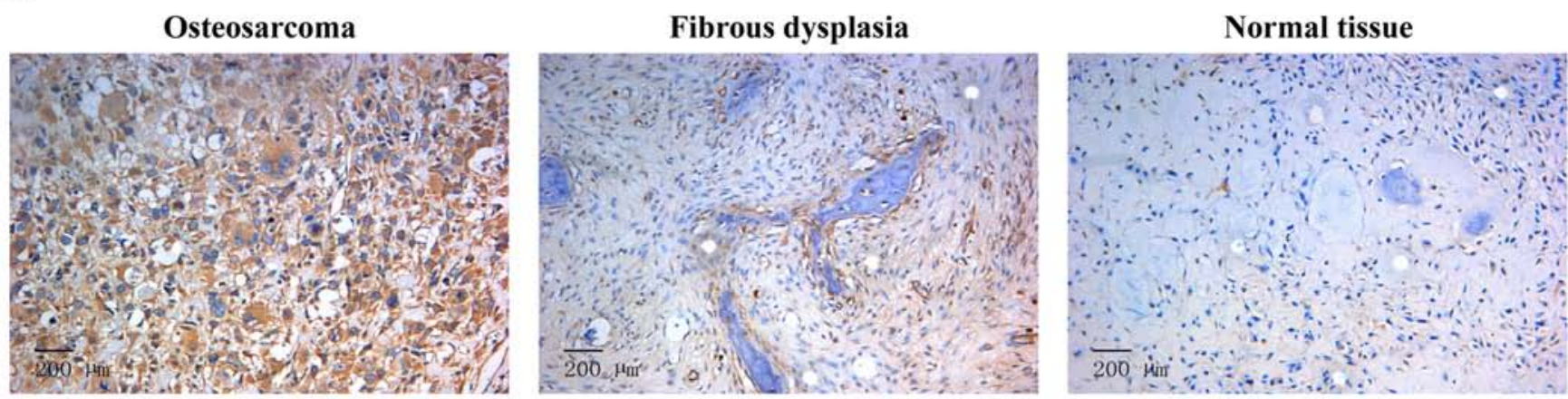

\section{B Alkaline phosphatase (ALP)}
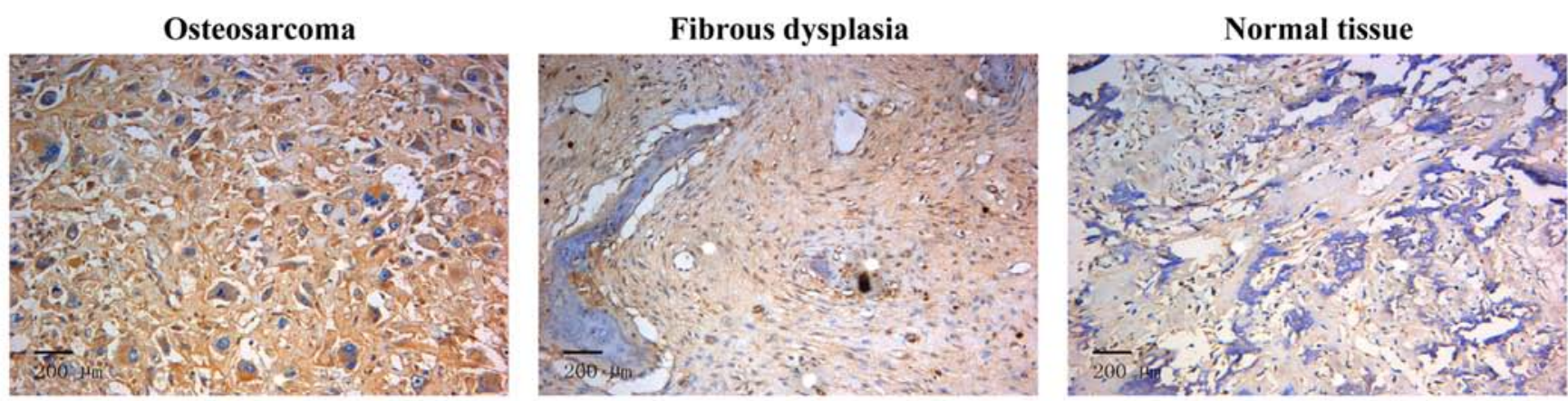

\section{Cysteine-rich angiogenic inducer 61 (CYR61)}
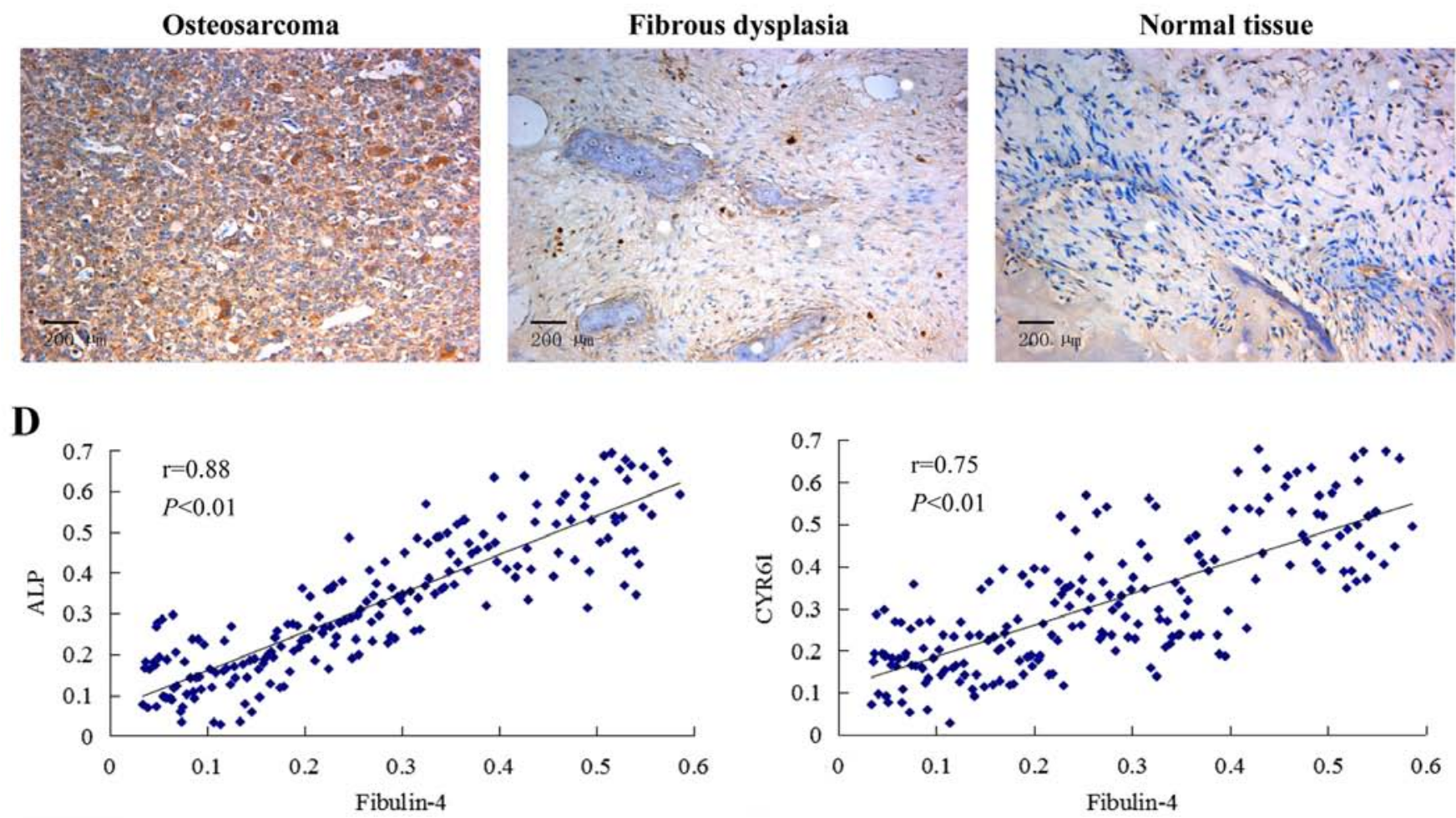

Figure 1. Expression levels of fibulin-4, CYR61 and ALP in human osteosarcoma tissues. (A) Fibulin-4 expression levels in osteosarcoma tissue, fibrous dysplasia tissue and normal tissue were measured by IHC. (B) ALP expression levels in osteosarcoma tissue, fibrous dysplasia tissue and normal tissue were measured by IHC. (C) CYR61 expression levels in osteosarcoma tissue, fibrous dysplasia tissue and normal tissue were measured by IHC. (D) According to the Pearson's product-moment correlation coefficient, the expression of fibulin-4 positively correlated with CYR61 and ALP, using photodensities detected by software Image-Pro Plus 6.0. (Magnification, x200).

to study the relationship between fibulin- 4 and the prognosis of patients with osteosarcoma. The data were analyzed with SPSS software version 13.0 (SPSS Inc., Chicago, IL, USA). $\mathrm{P}<0.05$ (two-sided) was considered statistically significant.

\section{Results}

The expression of fibulin-4, ALP and CYR61 in human osteosarcoma tissues. High fibulin-4 protein expression was 
Table I. Protein expression of fibulin-4 in human osteosarcoma tissues.

\begin{tabular}{|c|c|c|c|c|c|c|c|}
\hline & \multirow[b]{2}{*}{$\mathrm{N}$} & \multicolumn{2}{|c|}{$\begin{array}{l}\text { Fibulin-4 low } \\
\qquad(-/+)\end{array}$} & \multicolumn{2}{|c|}{$\begin{array}{l}\text { Fibulin-4 high } \\
\qquad(++/+++)\end{array}$} & \multirow[b]{2}{*}{$\chi^{2}$} & \multirow[b]{2}{*}{ P-value } \\
\hline & & $\mathrm{n}$ & $\%$ & $\mathrm{n}$ & $\%$ & & \\
\hline Normal tissue & 60 & 55 & 91.7 & 5 & 8.3 & 101.5 & $<0.01$ \\
\hline Fibrous dysplasia & 80 & 62 & 77.5 & 18 & 22.5 & & \\
\hline Osteosarcoma & 150 & 38 & 25.3 & 112 & 74.7 & & \\
\hline Pathological type & & & & & & 0.136 & $>0.05$ \\
\hline Fibroblastic osteosarcoma & 54 & 13 & 24.1 & 41 & 75.9 & & \\
\hline Osteoblastic osteosarcoma & 52 & 13 & 25 & 39 & 75 & & \\
\hline Chondroblastic osteosarcoma & 44 & 12 & 27.3 & 32 & 72.7 & & \\
\hline Cell differentiation & & & & & & 24.75 & $<0.01$ \\
\hline High and intermediate & 78 & 33 & 42.3 & 45 & 57.7 & & \\
\hline Low & 72 & 5 & 6.9 & 67 & 93.1 & & \\
\hline Nodal status & & & & & & 36.63 & $<0.01$ \\
\hline Positive & 83 & 5 & 6 & 78 & 94 & & \\
\hline Negative & 67 & 33 & 49.3 & 34 & 50.7 & & \\
\hline
\end{tabular}

detected in osteosarcoma tissues, mainly in the stroma and in the osteosarcoma cell cytoplasm. However, fibulin-4 immunoreactivity was very low in most normal tissues (Fig. 1A). Similar results were obtained in the detection of ALP and CYR61. Compared to normal tissues, the expression levels of ALP and CYR61 in osteosarcoma tissues were significantly high (Fig. 1B and C). According to the Pearson's product-moment correlation coefficient, the expression of CYR61 and ALP vs. fibulin-4 exhibited strong positive correlations (Fig. 1D). The expression of fibulin-4 in the extracellular matrix was found to be much less than that in the cancer cell cytoplasm, which probably was due to that fibulin-4 has an important role in development and integrity of extracellular matrices (3), loss of fibulin- 4 in the extracellular matrix would reduce the stability of extracellular matrix and promote cancer cell invasion and metastasis. Moreover, high fibulin-4 expression was positively associated with low differentiation and lymph node metastasis (Table I). Similar results were also observed in the qRT-PCR experiment. High fibulin-4 mRNA expression was observed in osteosarcoma tissues and was correlated with low tumor differentiation and positive nodal metastasis (Table II). Survival analysis was performed by Kaplan-Meier analysis. This result showed that patients with high fibulin-4 expression had poorer prognosis compared to those with low fibulin-4 expression (log rank, $\mathrm{P}<0.01$; Fig. 2A).

Establishment of highly invasive and low invasive subclones. Using the single cell cloning technique, 31 subclones were obtained from MG63 cells. The subclone MG63-1, which had the highest migration rate $(19.59 \pm 0.56 \mu \mathrm{m} / \mathrm{sec})$ showed higher proliferative and invasive abilities, compared to the subclone MG63-31, which showed the lowest migration rate $(7.68 \pm 0.13 \mu \mathrm{m} / \mathrm{sec})$. In vivo, the subcutaneous tumor formation rate for the highly invasive subclone group was $100 \%$, and was accompanied by rapid tumor growth. However, the tumor
Table II. mRNA expression of fibulin-4 in human osteosarcoma tissues.

\begin{tabular}{|c|c|c|c|}
\hline & $\mathrm{N}$ & $\begin{array}{c}\text { Fibulin- } 4 \text { mRNA } \\
\text { Normalized to } \\
\beta \text {-actin reference }\end{array}$ & P-value \\
\hline Normal tissue & 60 & $0.0114 \pm 0.0013$ & \\
\hline Fibrous dysplasia & 80 & $0.0345 \pm 0.0032$ & \\
\hline Osteosarcoma & 150 & $0.0996 \pm 0.0094$ & $<0.05$ \\
\hline Pathology type & & & $>0.05$ \\
\hline Fibroblastic osteosarcoma & 54 & $0.0847 \pm 0.0075$ & \\
\hline Osteoblastic osteosarcoma & 52 & $0.0913 \pm 0.0063$ & \\
\hline Chondroblastic osteosarcoma & 44 & $0.0891 \pm 0.0082$ & \\
\hline Cell differentiation & & & $<0.05$ \\
\hline High and medium & 78 & $0.0487 \pm 0.0037$ & \\
\hline Low & 72 & $0.0986 \pm 0.0091$ & \\
\hline Nodal status & & & $<0.05$ \\
\hline Positive & 83 & $0.0958 \pm 0.0087$ & \\
\hline Negative & 67 & $0.0397 \pm 0.0053$ & \\
\hline
\end{tabular}

formation rate of the low invasive subclone group was only approximately $50 \%$, with very slow tumor growth. The tumor volume for MG63-1 was 479.82 $\pm 34.31 \mathrm{~mm}^{3}$, much larger than that formed by MG63-31 (35.91 $\left.\pm 3.73 \mathrm{~mm}^{3}, \mathrm{P}<0.01\right)$. These results are shown in Fig. 2.

Different proliferation and invasion abilities of human osteosarcoma cell lines and the normal osteoblastic cell line. Compared to the normal osteoblastic cell line hFOB, 
A

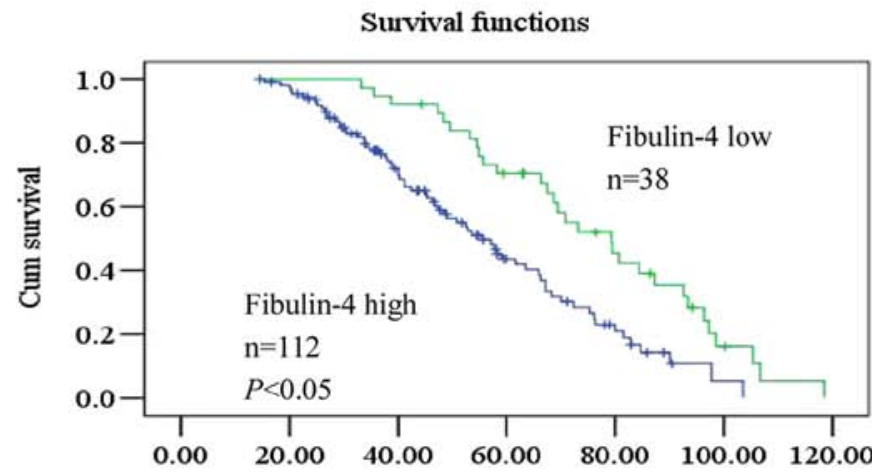

Time (months)

B

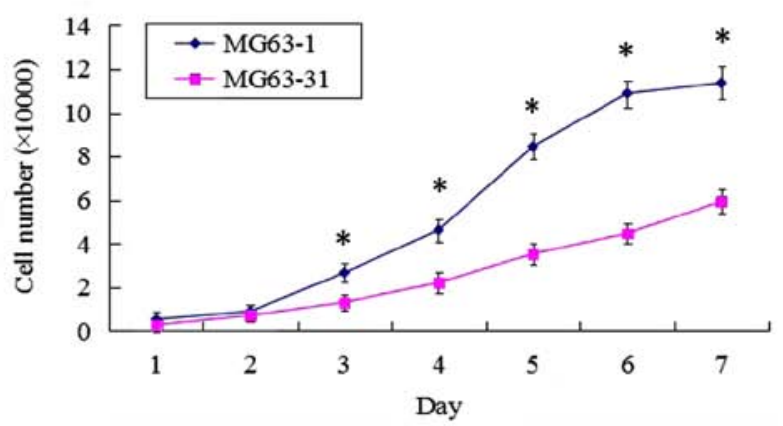

D

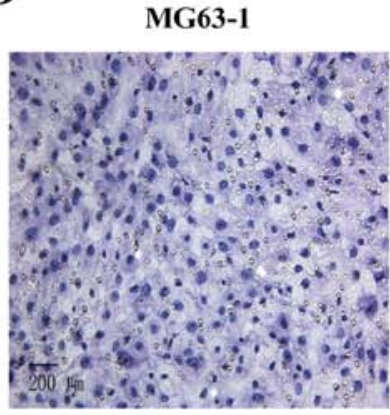

$\mathbf{F}$

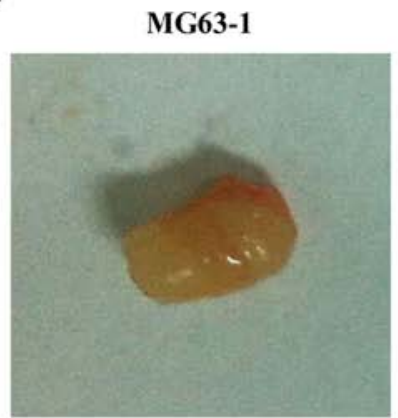

MG63-31

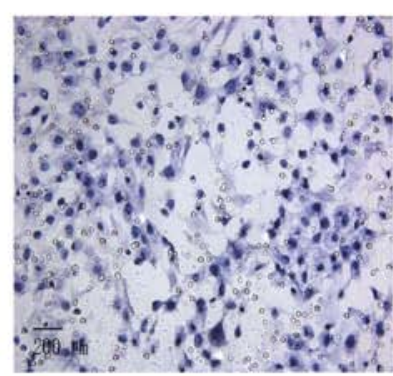

MG63-31

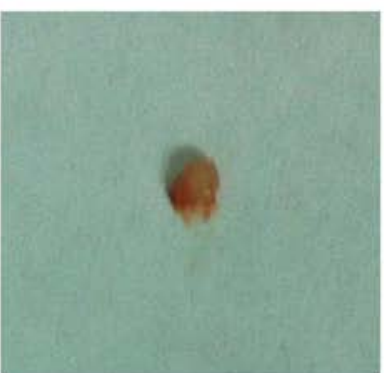

C

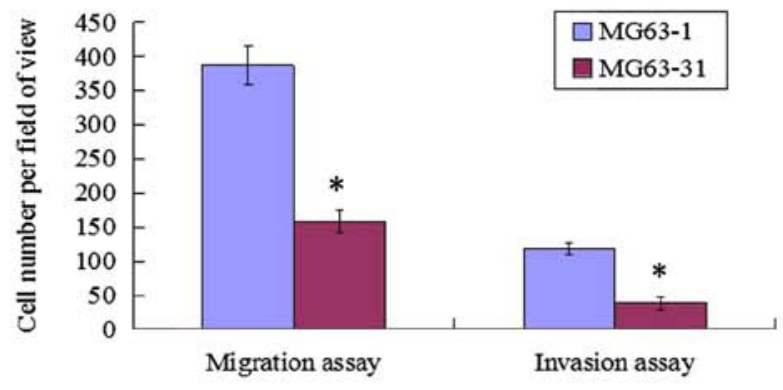

$\mathbf{E}$
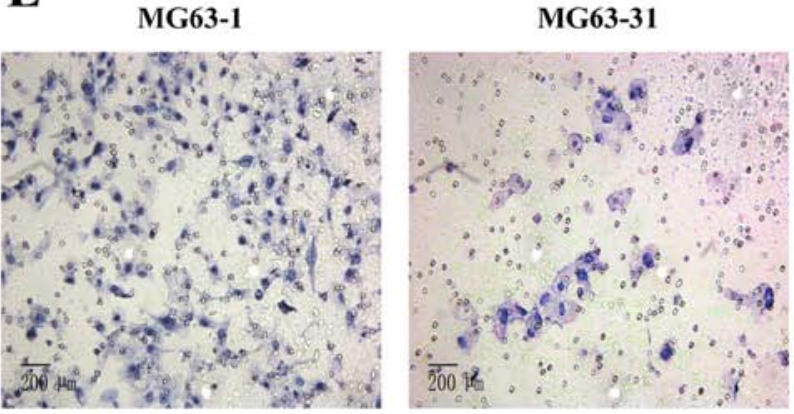

G

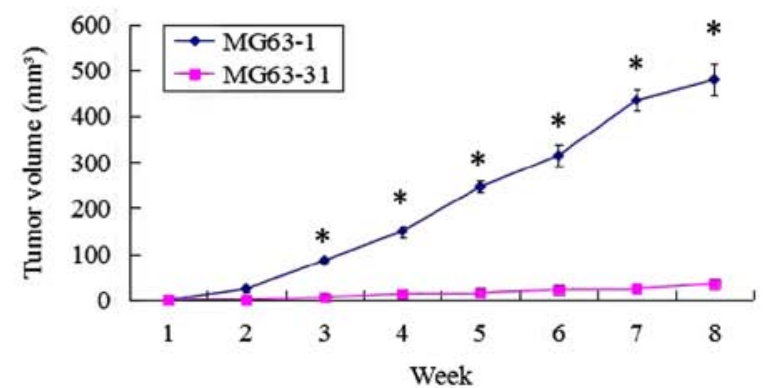

Figure 2. Kaplan-Meier analysis and the establishment of high invasive subclone and low invasive subclone. (A) Kaplan-Meier curve showing overall survival. Patients with high fibulin-4 expression (blue line) had a much worse prognosis than those with low fibulin-4 expression (green line). (B) By growth curves, the high invasive subclone had higher proliferative abilities than the low invasive subclone. (C) By cell migration and invasion assays using Boyden chambers, the average counts of migrating and invading cells of high invasive subclone were both much higher than those of low invasive subclone. (D) The images of cells crossing PVPF filters without Matrigel were examined by cell migration assay. (E) The images of cells crossing PVPF filters with Matrigel were examined by cell invasion assay. (F) The tumor volumes formed by high invasive subclone were much larger than those formed by low invasive subclone after subcutaneous inoculation. (G) Tumor growths of high invasive subclone and low invasive subclone were observed continuously for 2 months. (Magnification, $\mathrm{x} 200$ ). ${ }^{*} \mathrm{P}<0.05$.

the human osteosarcoma cell lines U-2OS and MG63 showed stronger proliferative abilities (Fig. 3A). In the soft agar colony formation assay, the number of colonies formed by MG63 and U-2OS was also significantly greater than that formed by hFOB (Fig. 3B). In the cell migration and Matrigel invasion assays, the average counts of migrating and invading MG63 
A

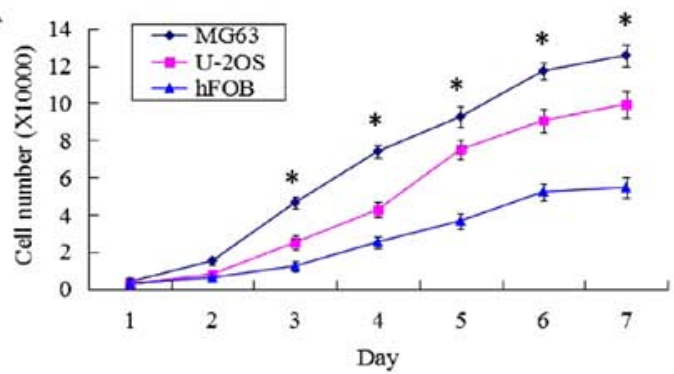

C

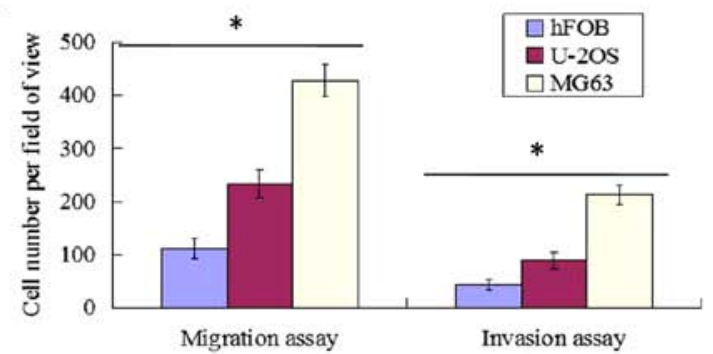

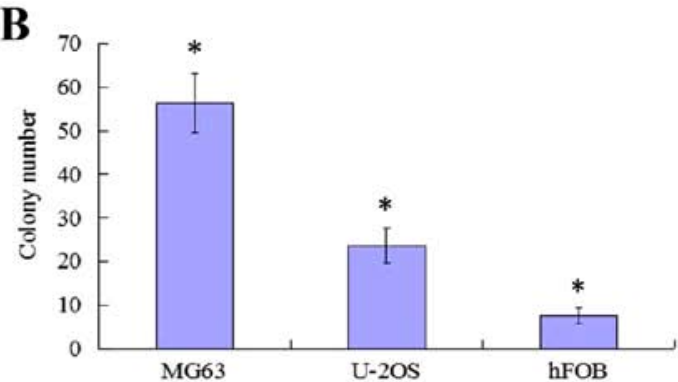

D

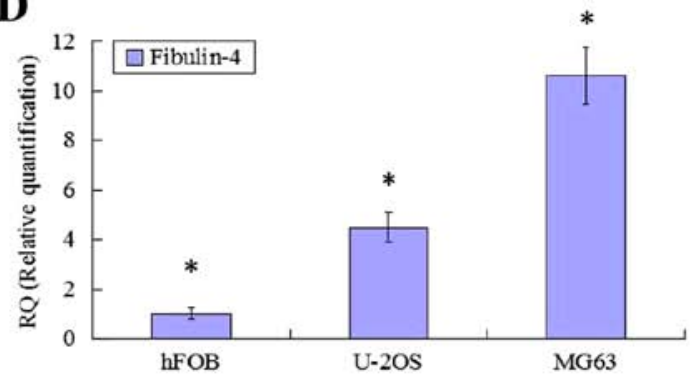

E

hFOB

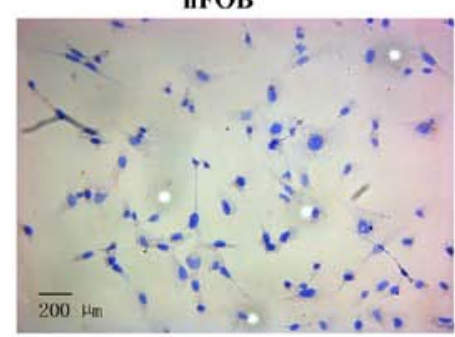

F

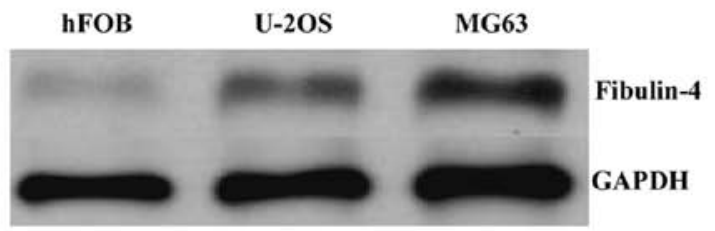

H

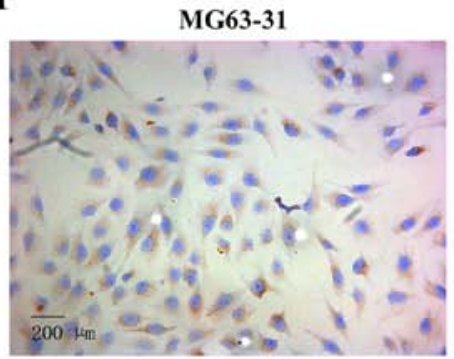

U-2OS
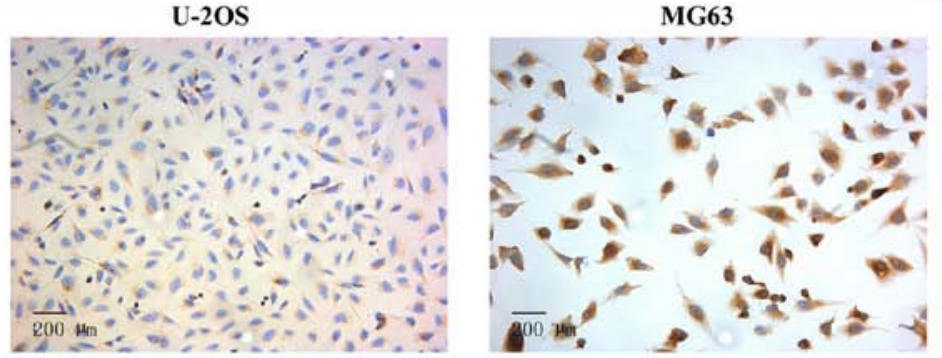

G

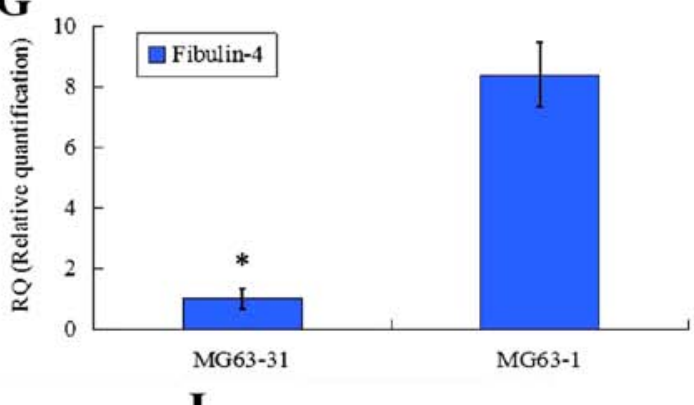

I
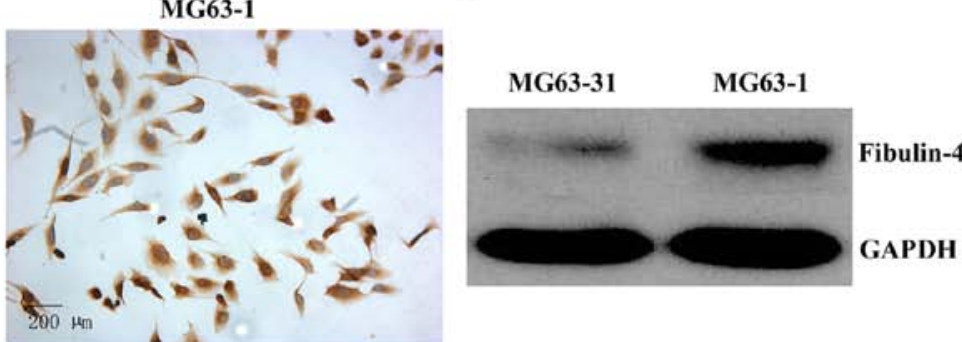

Figure 3. Fibulin-4 expression levels in differently invasive osteosarcoma cell lines and subclones. (A) By growth curves, the human osteosarcoma cell lines U-2OS and MG63 had stronger proliferative abilities than normal osteoblastic cell line hFOB. (B) By soft agar colony formation assay, the number of colonies formed by MG63 and U-2OS was also significantly greater than that formed by hFOB. (C) By cell migration and invasion assays using Boyden chambers, the average counts of migrating and invading cells of human osteosarcoma cell lines MG63 and U-2OS were both much higher than those of normal osteoblastic cell line hFOB. Fibulin-4 expression levels in human osteosarcoma cell lines MG63 and U-2OS and normal osteoblastic cell line hFOB were measured by real-time q-RT-PCR (D), ICC staining (E) and western blotting (F). Fibulin-4 expression levels in high invasive subclone and low invasive subclone were measured by real-time q-RT-PCR (G), ICC staining (H) and western blotting (I). (Magnification, x200). ${ }^{*} \mathrm{P}<0.05$.

and U-2OS cells were both much higher than those of hFOB (Fig. 3C). Upon comparing the two osteosarcoma cell lines, we found that MG63 had stronger proliferation and invasion abilities than those of U-2OS.
Fibulin-4 expression in human osteosarcoma cell lines and in differently invasive subclones. As shown in Fig. 3D-F, fibulin-4 was very weakly expressed in the normal osteoblastic cell line hFOB compared to in the human osteosarcoma cell 
A

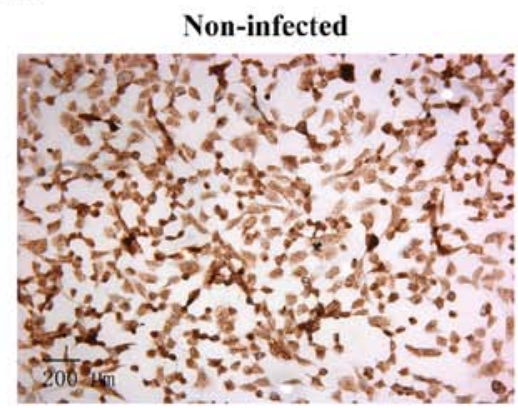

B

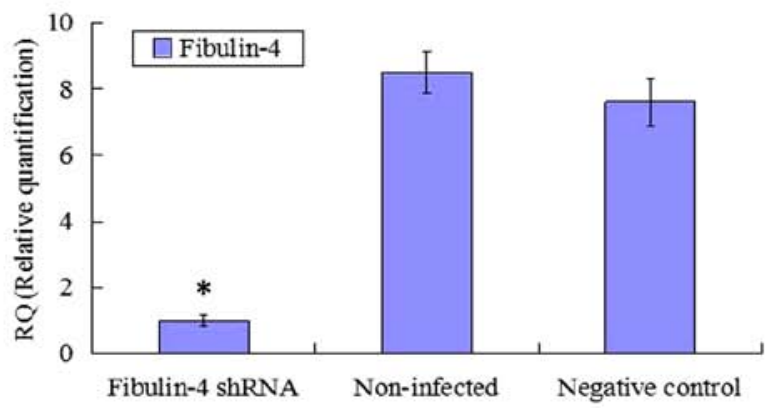

HOS

Negative control

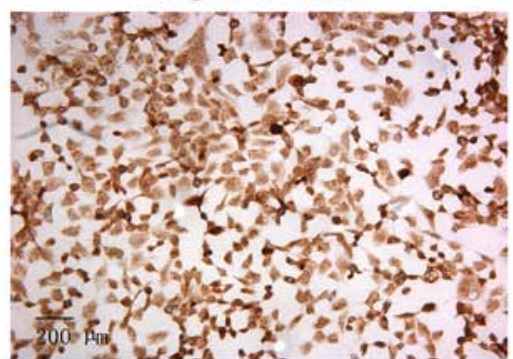

C
Fibulin-4 shRNA

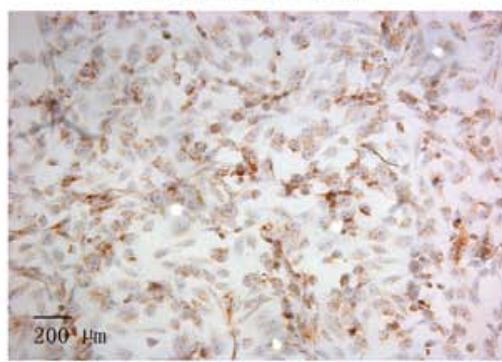

HOS
D

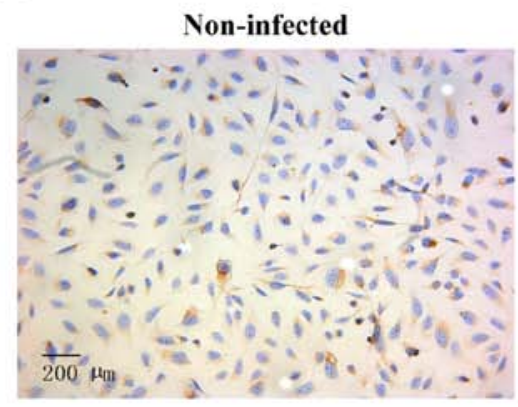

$\mathbf{E}$

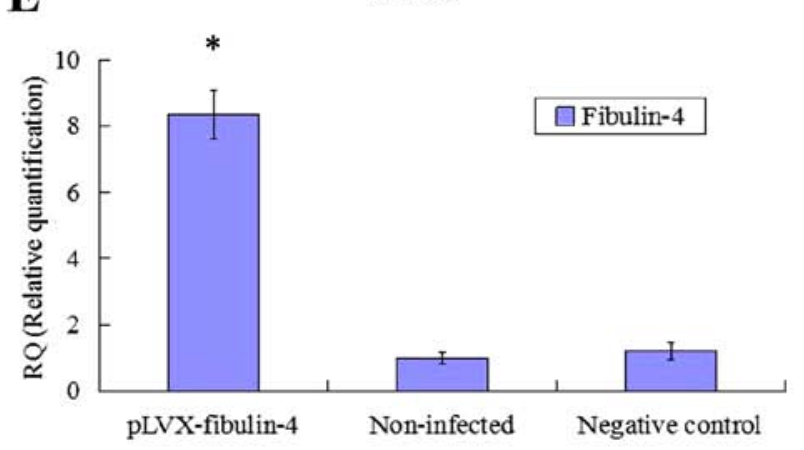

U-20S

Negative control

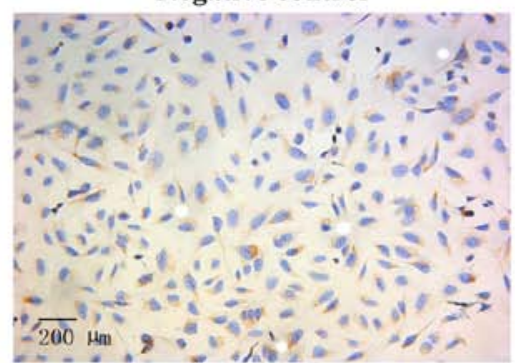

$\mathbf{F}$

F

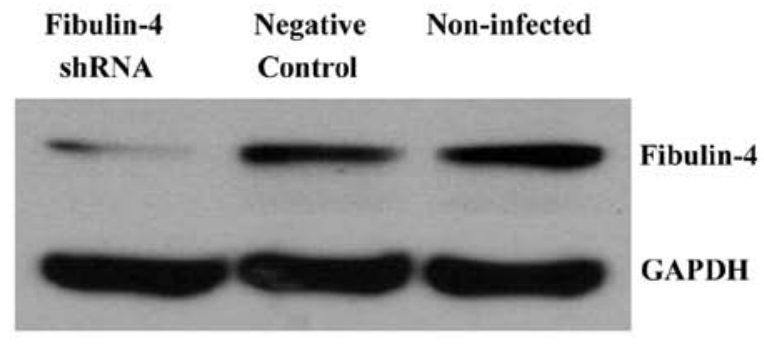

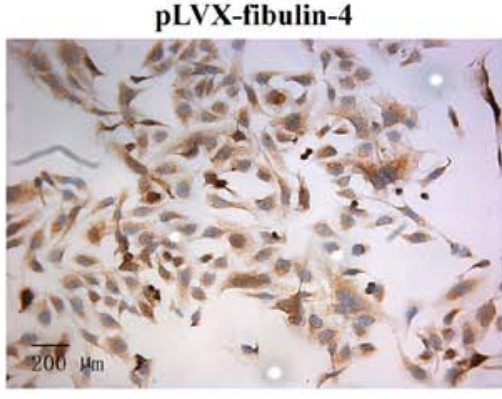

U-2OS

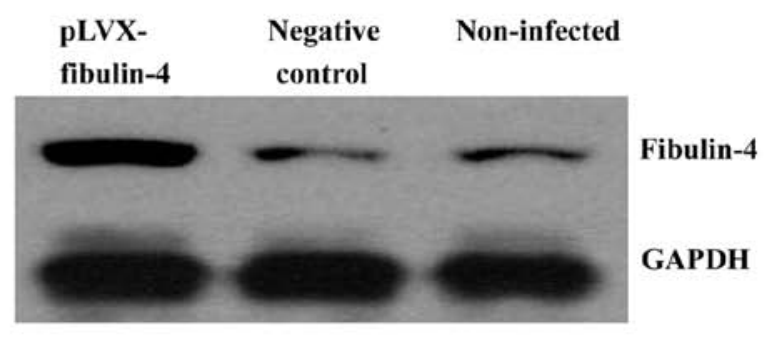

Figure 4. Identification of the transfection efficiencies after lentivirus-mediated transfection. After RNA interference, fibulin-4 mRNA and protein expression levels in non-infected HOS cells, control shRNA infected HOS cells and fibulin-4 shRNA infected HOS cells were measured by (A) ICC staining, (B) real-time q-RT-PCR and (C) western blotting, which indicated high efficiency in the RNA interference experiments. After overexpression transfection, fibulin-4 mRNA and protein expression levels in non-infected U-2OS cells, control shRNA infected U-2OS cells and pLVX-fibulin-4 infected U-2OS cells were measured by (D) ICC staining, (E) real-time q-RT-PCR and (F) western blotting, which indicated high efficiency in the overexpression transfection experiments. (Magnification, $\mathrm{x} 200)$. ${ }^{*} \mathrm{P}<0.05$.

lines MG63 and U-2OS. The strongest fibulin-4 expression was detected in MG63, which showed the highest proliferation and invasion abilities. Similar results were also observed upon comparing subclones with differing invasive abilities (Fig. 3G-I). Compared with the low invasive subclone MG63-31, high fibulin-4 expression was detected in the highly invasive subclone MG63-1. These results indicate that high fibulin-4 expression might be positively associated with the proliferative and invasive abilities of osteosarcoma cells.

Identification of downregulated and upregulated fibulin-4 expression in lentivirus transfection systems. To further 
A

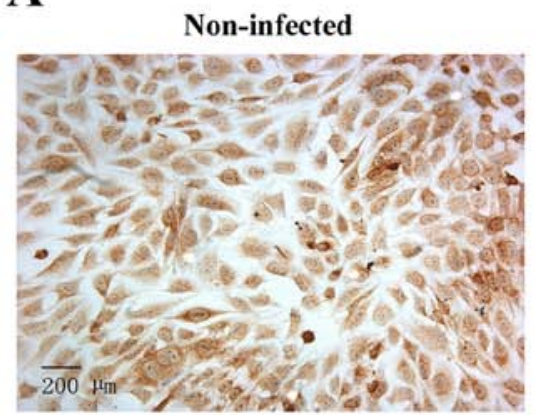

B

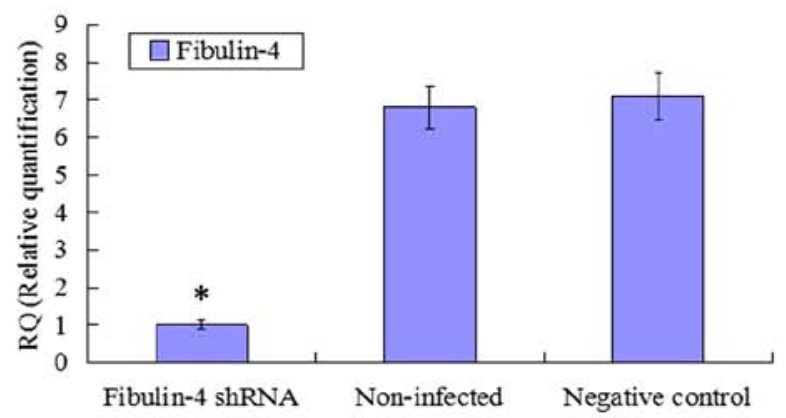

MG63-1

Negative control

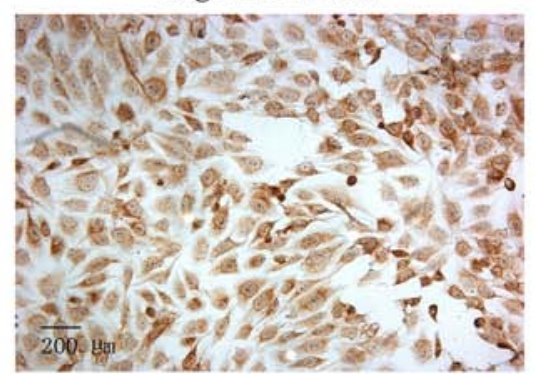

C
Fibulin-4 shRNA

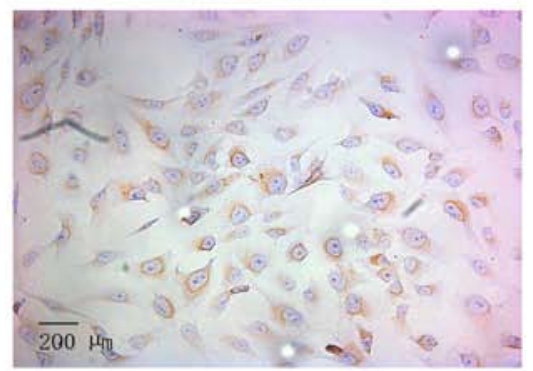

MG63-1
D

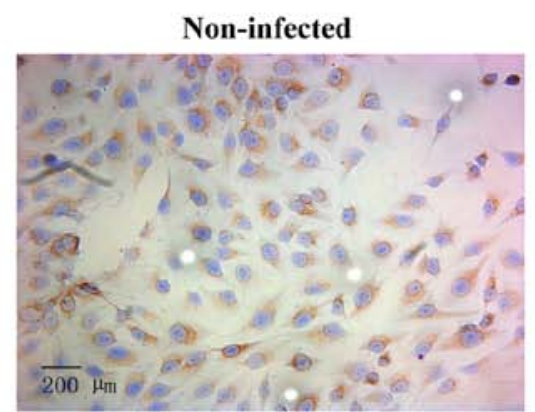

E

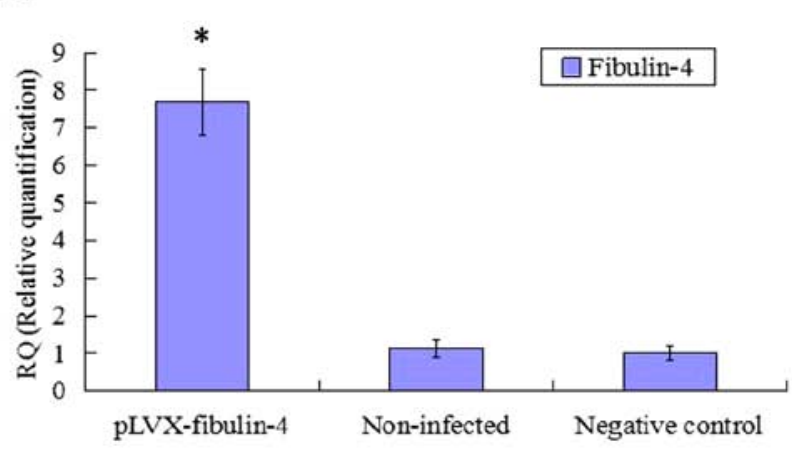

MG63-31

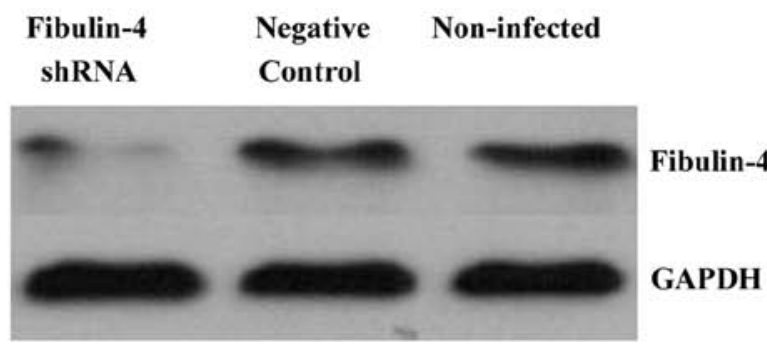

Negative control

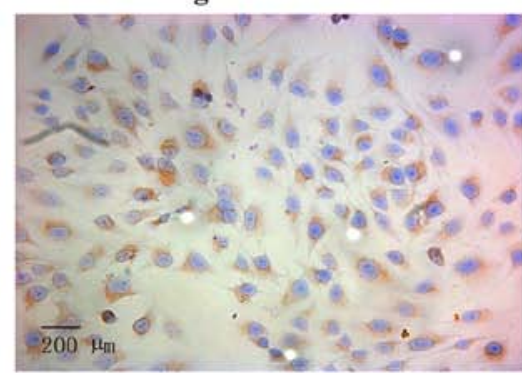

F
pLVX-fibulin-4

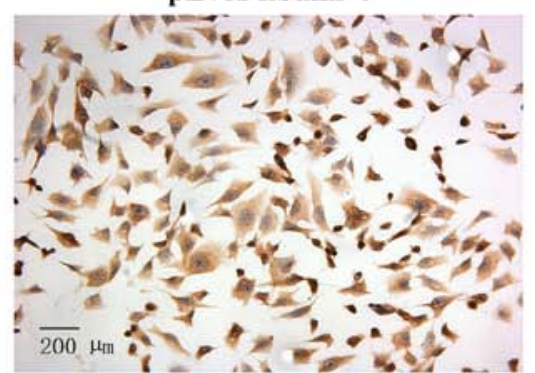

MG63-31

$\begin{array}{lll}\text { pLVX- } & \text { Negative } & \text { Non-infected } \\ \text { fibulin-4 } & \text { control }\end{array}$

control

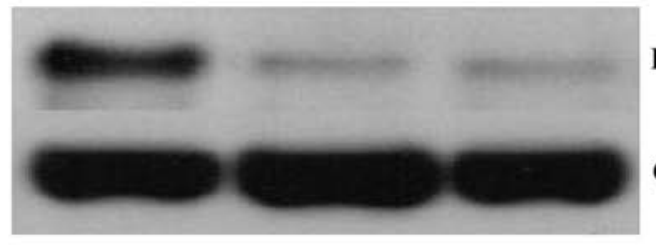

Fibulin-4

GAPDH

Figure 5. Identification of downregulated and upregulated fibulin-4 expression in lentivirus transfection systems. After RNA interference, fibulin-4 mRNA and protein expression levels in non-infected MG63-1 cells, control shRNA infected MG63-1 cells and fibulin-4 shRNA infected MG63-1 cells were measured by (A) ICC staining, (B) real-time q-RT-PCR and (C) western blotting, which indicated high efficiency in the RNA interference experiments. After overexpression transfection, fibulin-4 mRNA and protein expression levels in non-infected MG63-31 cells, control shRNA infected MG63-31 cells and pLVX-fibulin-4 infected MG63-31 cells were measured by (D) ICC staining, (E) real-time q-RT-PCR and (F) western blotting, which indicated high efficiency in the overexpression transfection experiments. (Magnification, $\mathrm{x} 200){ }^{*} \mathrm{P}<0.05$.

investigate the potential role of fibulin-4 in osteosarcoma cell proliferation and invasion, we decreased the expression of fibulin-4 in the highly invasive osteosarcoma cell line HOS and subclone MG63-1, and increased fibulin-4 expression in the low invasive osteosarcoma cell line U-2OS and subclone
MG63-31, by lentivirus transfection. After viral infection, real-time q-RT-PCR, western blotting, and ICC were used to confirm the altered expression of fibulin- 4 at both mRNA and protein levels, indicating the high efficiency of the lentivirus transfections (Figs. 4 and 5). 
A

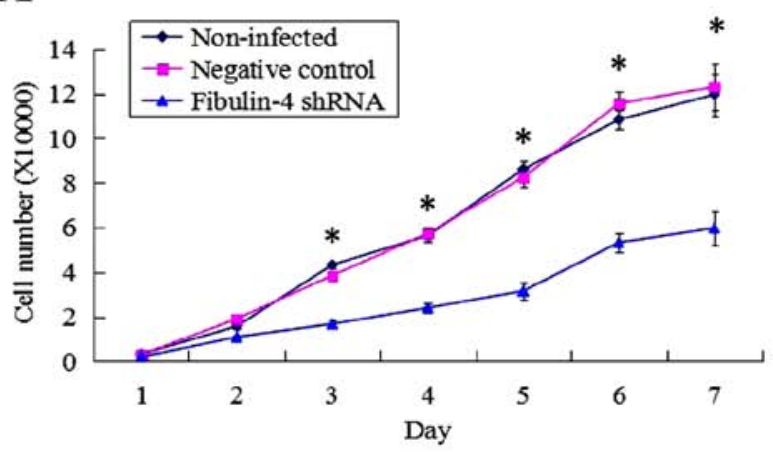

B

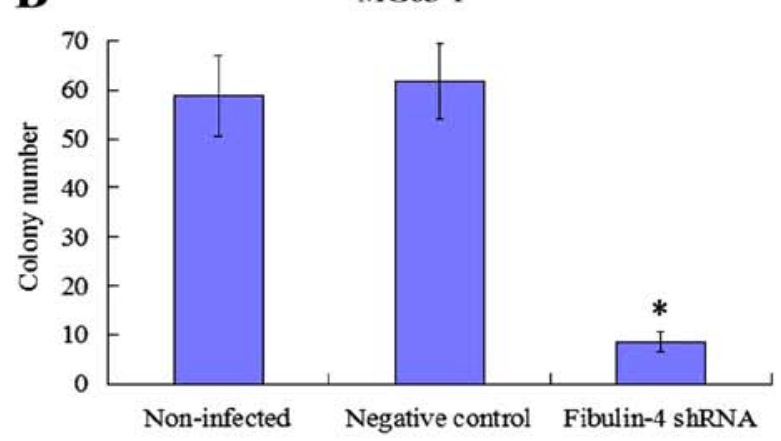

MG63-31

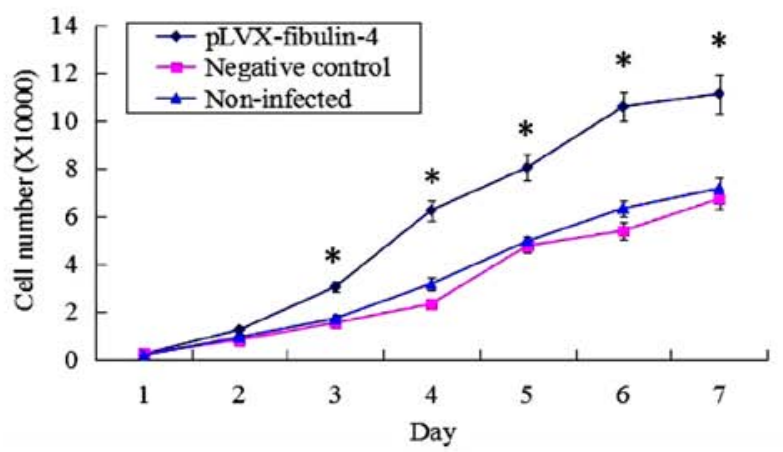

MG63-31

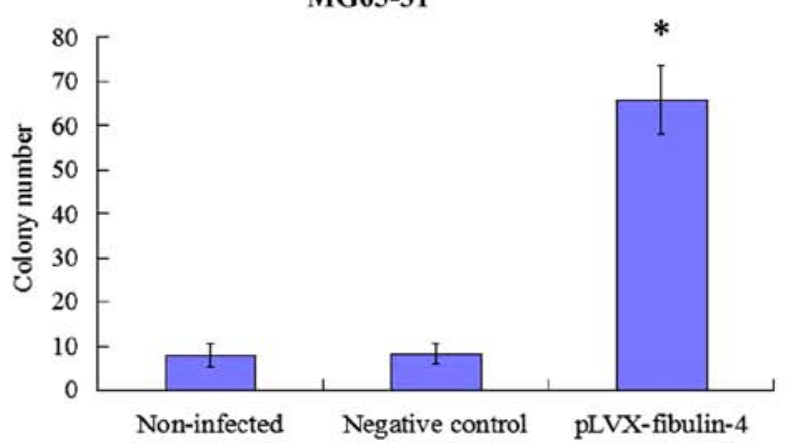

C

MG63-1

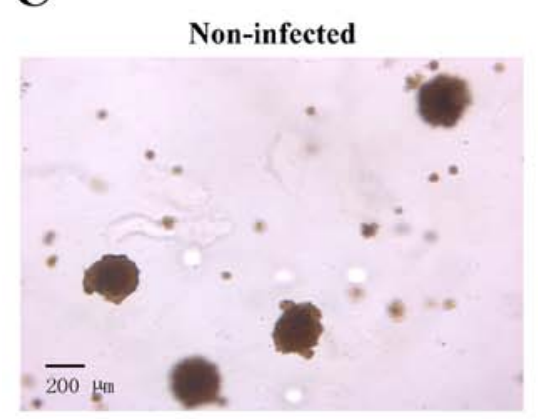

D

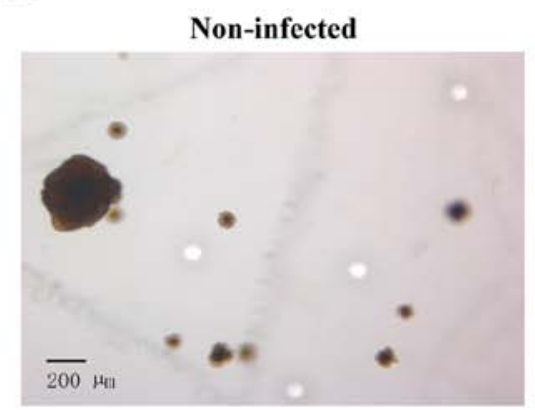

Negative control

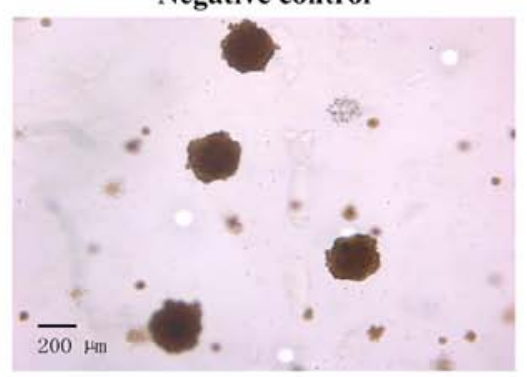

MG63-31

Negative control

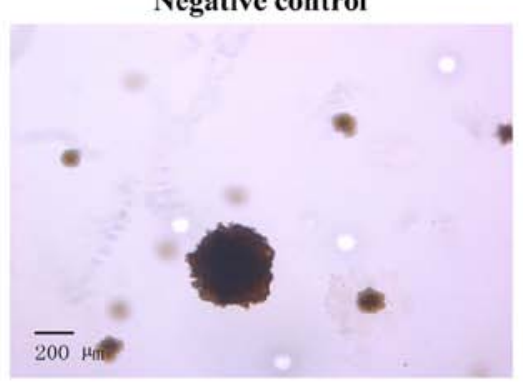

Fibulin-4 shRNA

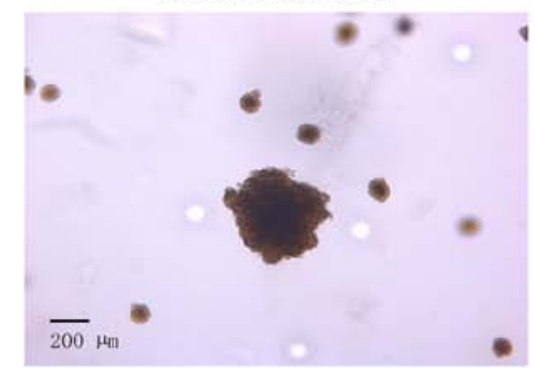

pLVX-fibulin-4

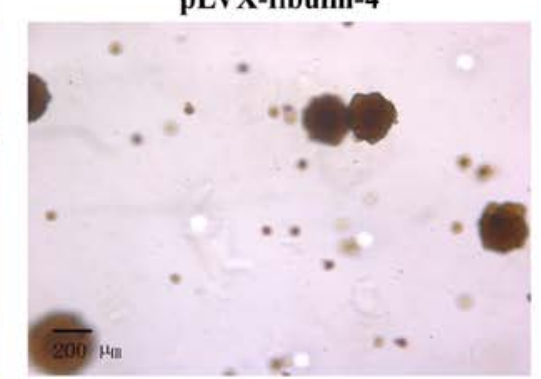

Figure 6. Effect of fibulin-4 knockdown and overexpression on osteosarcoma cell proliferation and colony formation abilities. (A) By growth curves, downregulated fibulin-4 markedly inhibited cell proliferation of the highly invasive subclone MG63-1, whereas upregulated fibulin-4 significantly promoted cell proliferation of the low invasive subclone MG63-31. (B) By soft agar colony formation assay, the colony forming efficiencies of fibulin-4-silenced cells were decreased, and conversely, upregulation of fibulin-4 could increase the colony forming efficiency of the low invasive subclone. (C) The colony images of non-infected cells, control shRNA infected cells and fibulin-4 shRNA infected cells were examined by soft agar colony formation assay. (D) The colony images of non-infected cells, control shRNA infected cells and pLVX-fibulin-4 infected cells were examined by soft agar colony formation assay. (Magnification, x200). ${ }^{*} \mathrm{P}<0.05$.

Effect of fibulin-4 knockdown and overexpression on osteosarcoma cell proliferation. Downregulated fibulin-4 markedly inhibited cell proliferation of the highly invasive subclone MG63-1, whereas upregulated fibulin-4 significantly promoted cell proliferation of the low invasive subclone MG63-31 (Fig. 6A). In the soft agar colony formation assay, the colony forming efficiency of fibulin-4-silenced cells was decreased, and conversely, upregulation of fibulin-4 increased the colony 
A

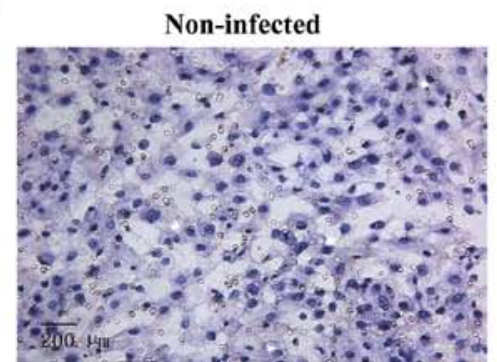

B

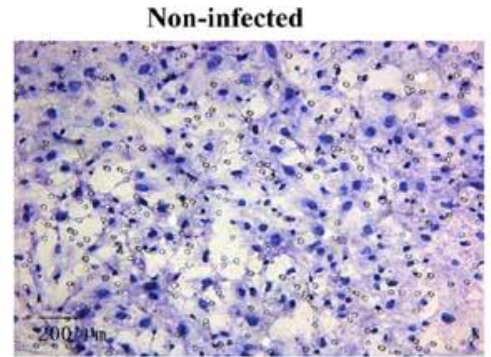

C

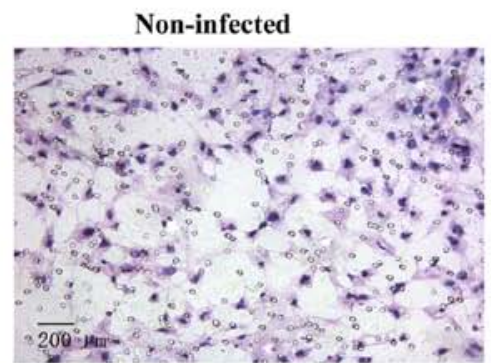

D

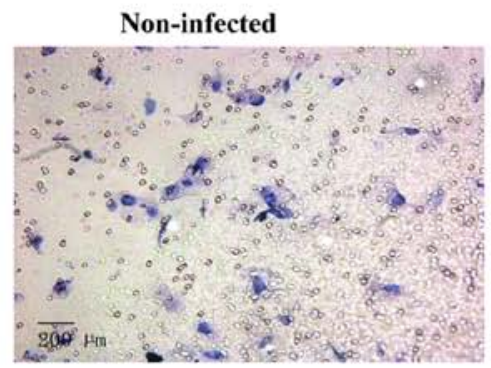

MG63-1

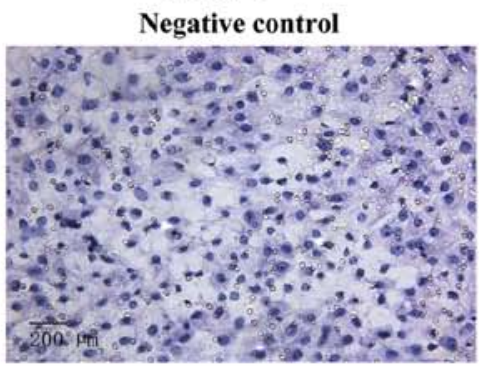

Negative control

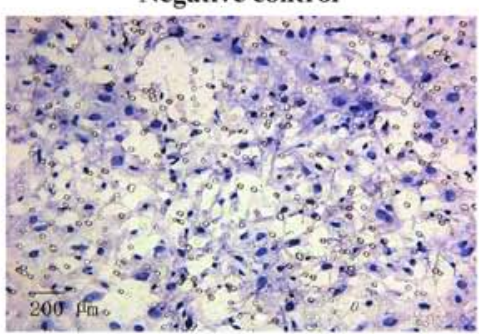

MG63-31

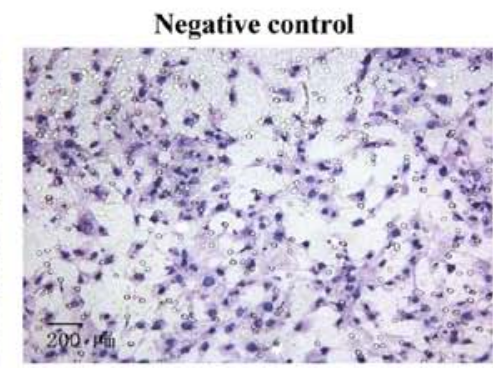

Negative control

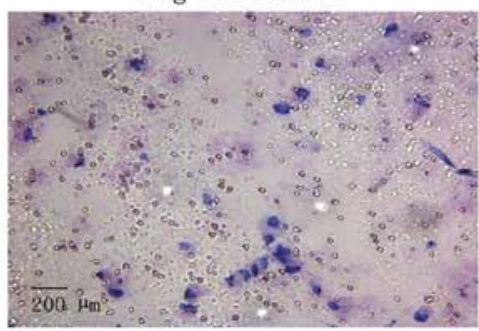

Fibulin-4 shRNA

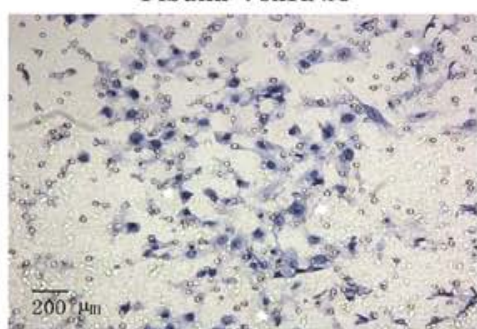

Fibulin-4 shRNA

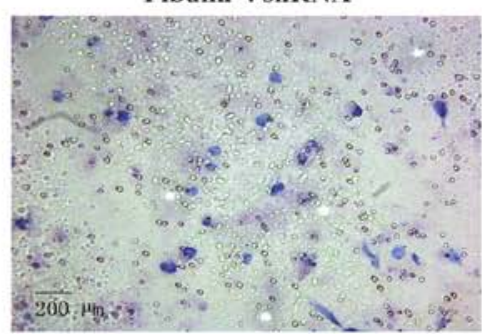

pLVX-fibulin-4

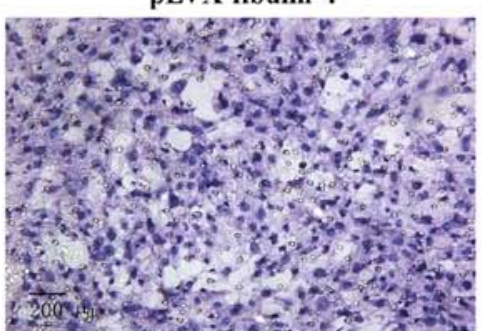

pLVX-fibulin-4

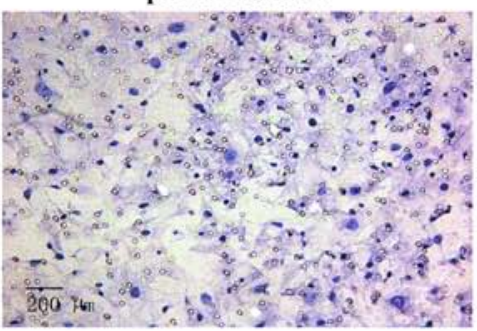

E
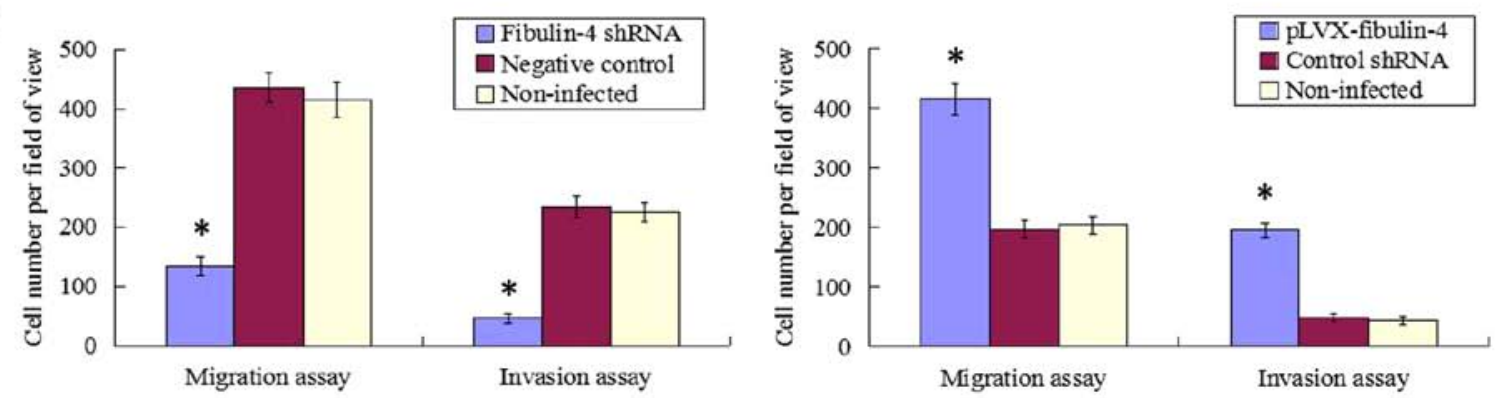

Figure 7. Effect of fibulin-4 knockdown and overexpression on the migration and invasion of the differently invasive osteosarcoma cell subclones. After RNA interference, cell migration images (A) and invasion images (B) of non-infected MG63-1 cells, control shRNA infected MG63-1 cells and fibulin-4 shRNA infected MG63-1 cells were measured by cell migration and invasion assays using Boyden chambers. After overexpression transfection, cell migration images (C) and invasion images (D) of non-infected MG63-31 cells, control shRNA infected MG63-31 cells and pLVX-fibulin-4 infected MG63-31 cells were measured by cell migration and invasion assays using Boyden chambers. (E) The average counts of migrating and invading fibulin-4 shRNA infected MG63-1 cells were much lower than those of the negative controls and non-infected groups; whereas, the average counts of migrating and invading pLVX-fibulin- 4 infected MG63-31 cells were much higher than those of the negative controls and the non-infected groups. (Magnification, x200). ${ }^{*} \mathrm{P}<0.05$.

forming efficiency of the low invasive subclone (Fig. 6B and C). No significant differences were observed in the noninfected and negative control groups.
Effect of fibulin-4 knockdown and overexpression on osteosarcoma cell migration and invasion. As shown in Figs. 7 and 8, fibulin-4 knockdown inhibited osteosarcoma cell 
$\mathbf{A}$

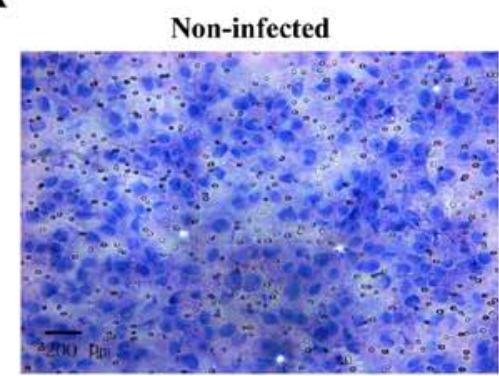

B

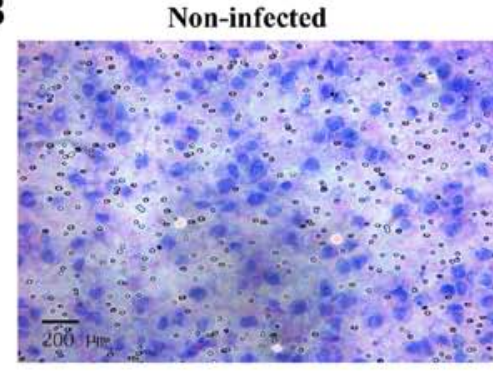

C

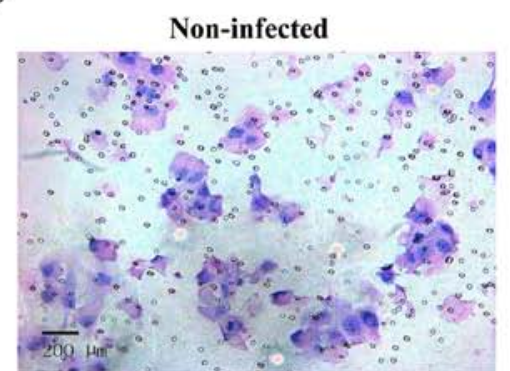

D

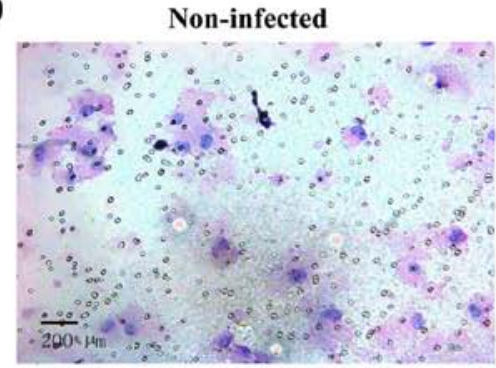

HOS

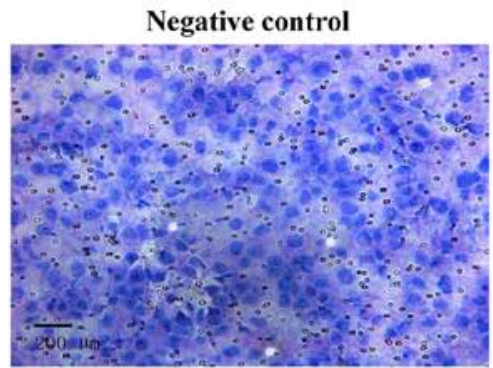

Negative control

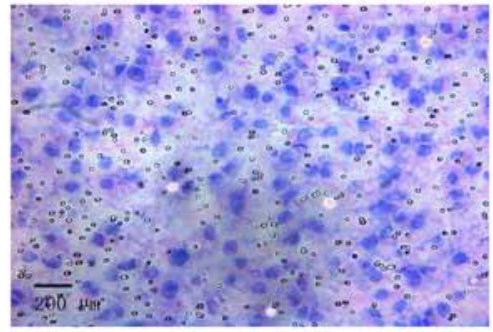

U-20S

Negative control

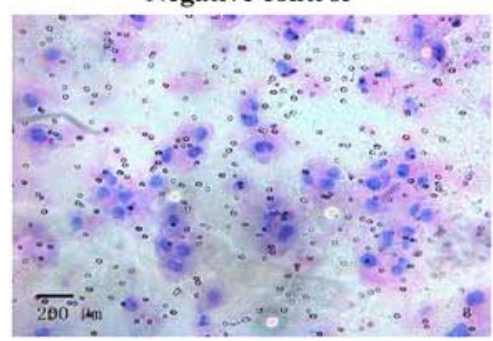

Negative control

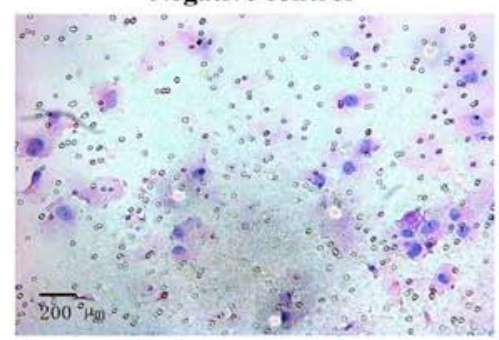

Fibulin-4 shRNA

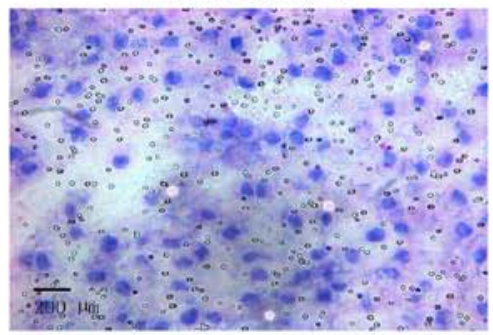

Fibulin-4 shRNA

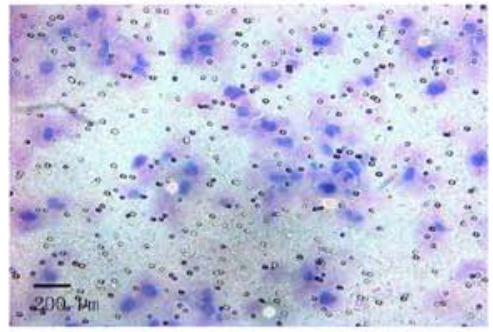

pLVX-fibulin-4

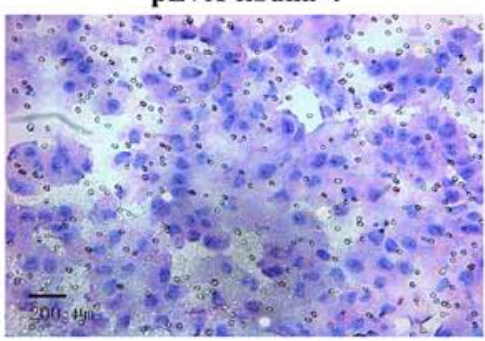

pLVX-fibulin-4

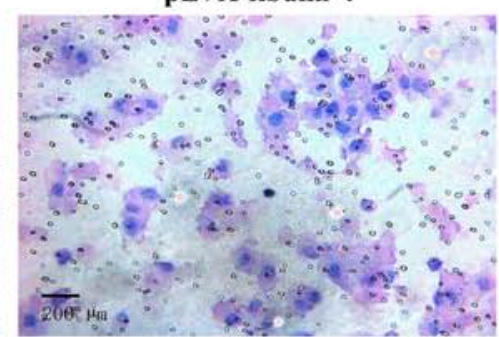

$\mathbf{E}$
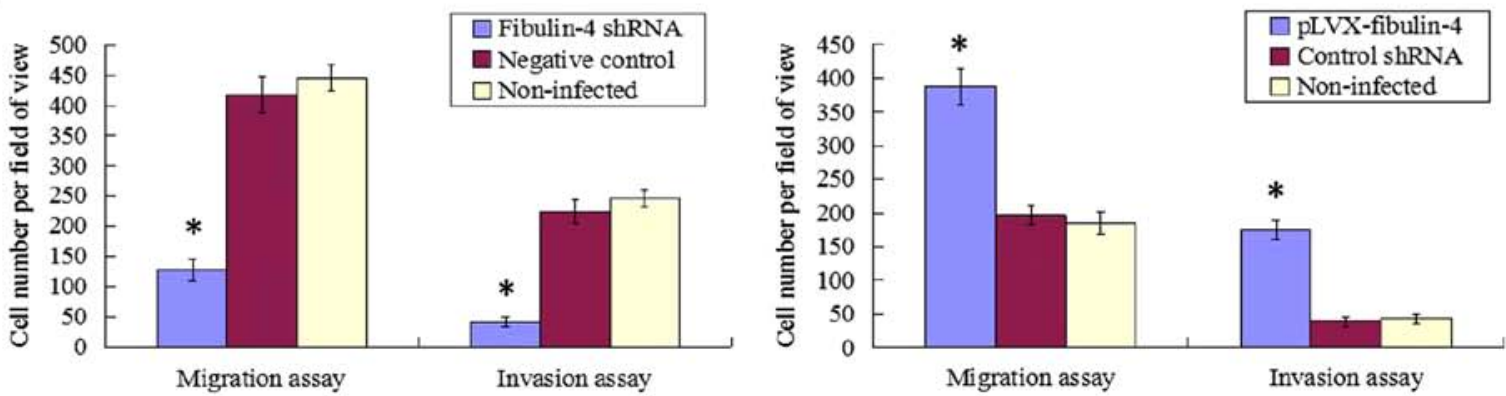

Figure 8. Effect of fibulin-4 knockdown and overexpression on osteosarcoma cell migration and invasion. After RNA interference, cell migration images (A) and invasion images (B) of non-infected HOS cells, control shRNA infected HOS cells and fibulin-4 shRNA infected HOS cells were measured by cell migration and invasion assays using Boyden chambers. After overexpression transfection, cell migration images (C) and invasion images (D) of non-infected U-2OS cells, control shRNA infected U-2OS cells and pLVX-fibulin-4 infected U-2OS cells were measured by cell migration and invasion assays using Boyden chambers. (E) The average counts of migrating and invading fibulin-4 shRNA infected HOS cells were much lower than those of the negative controls and non-infected groups; whereas, the average counts of migrating and invading pLVX-fibulin-4 infected U-2OS cells were much higher than those of the negative controls and the non-infected groups. (Magnification, $\mathrm{x} 200$ ). ${ }^{*} \mathrm{P}<0.05$.

invasion and migration. The average counts of migrating and invading fibulin-4 shRNA infected cells were much lower than those of the negative controls and non-infected groups $(\mathrm{P}<0.05)$, whereas, fibulin-4 overexpression promoted the 

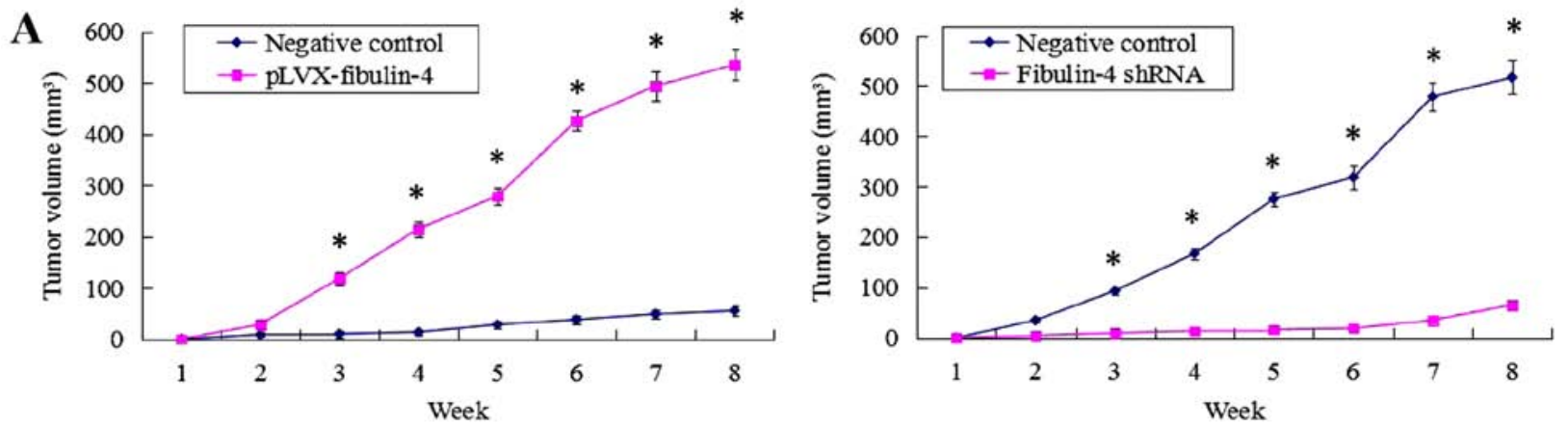

B

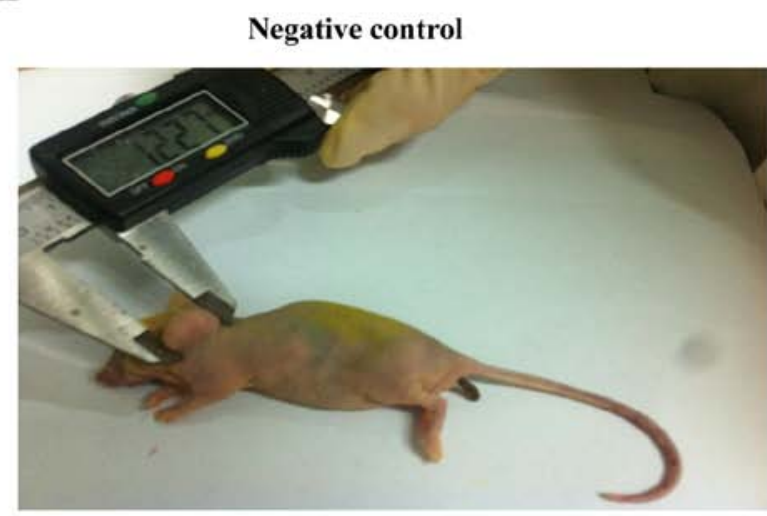

MG63-1

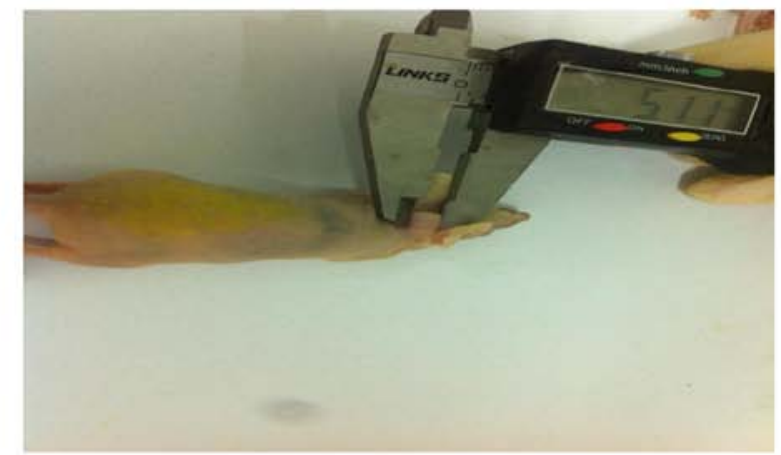

C

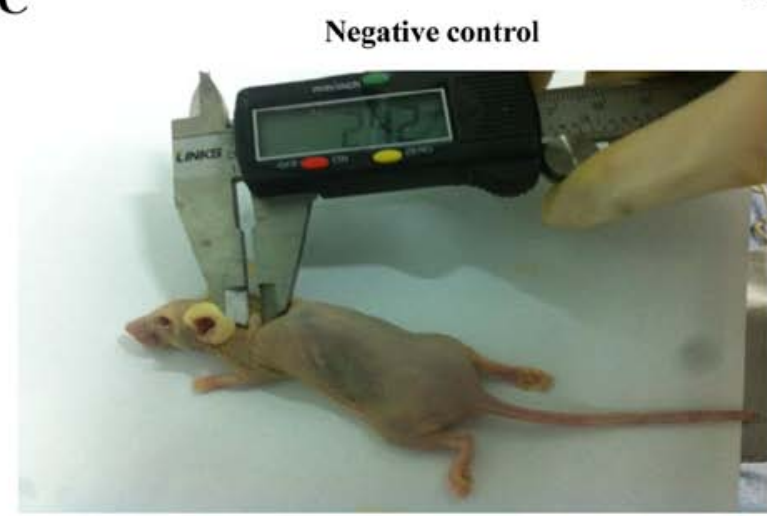

MG63-31

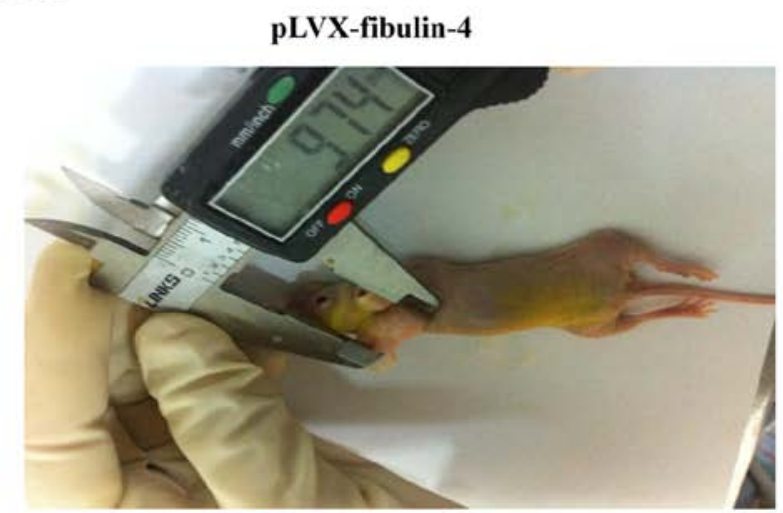

Figure 9. Effects of fibulin-4 knockdown and overexpression on tumor growth in a xenograft model. (A) Tumor growths in lentivirus transfection system was observed continuously for 8 weeks. (B) The average volumes of the tumors formed in the fibulin- 4 shRNA infected group were much smaller than those formed by the negative control MG63-1. (C) The average volumes of the tumors formed by pLVX-fibulin- 4 infected cells were much larger than those formed by the negative control MG63-31. ${ }^{*} \mathrm{P}<0.05$.

invasion and migration of osteosarcoma cells. The average counts of migrating and invading pLVX-fibulin- 4 infected cells were much higher than those of the negative controls and the non-infected groups $(\mathrm{P}<0.05)$. There were no significant differences between the negative controls and non-infected groups.

Effects of fibulin-4 knockdown and overexpression on tumor growth in a xenograft model. The fibulin-4 shRNA infected cells, pLVX-fibulin-4 infected cells, negative control MG63-1, and negative control MG63-31 were each inoculated subcutaneously in 5 nude mice, respectively. The tumor formation rate of the negative control MG63-1 was $100 \%$, whereas the tumor formation rate in the fibulin- 4 shRNA infected group was only $60 \%$. Moreover, the average volumes of the tumors formed in the fibulin-4 shRNA infected group were much lower than those formed by the negative control MG63-1. Fibulin-4 knockdown inhibited tumor formation in nude mice. Simultaneously, fibulin-4 overexpression promoted tumor growth in nude mice. The tumor formation rate of pLVX-fibulin-4 infected cells was $100 \%$, whereas the tumor formation rate of the negative control MG63-31 was only $40 \%$. Moreover, the average volumes of the tumors formed by pLVX-fibulin-4 infected cells were much higher than those formed by the negative control MG63-31 (Fig. 9).

Effects of fibulin-4 on key epithelial-mesenchymal transition genes, CYR61 and ALP. CYR61 and ALP were significantly associated with osteosarcoma development and progression. Therefore, we wondered if fibulin- 4 knockdown and upregulation affected these genes. As shown in Fig. 10, 
A

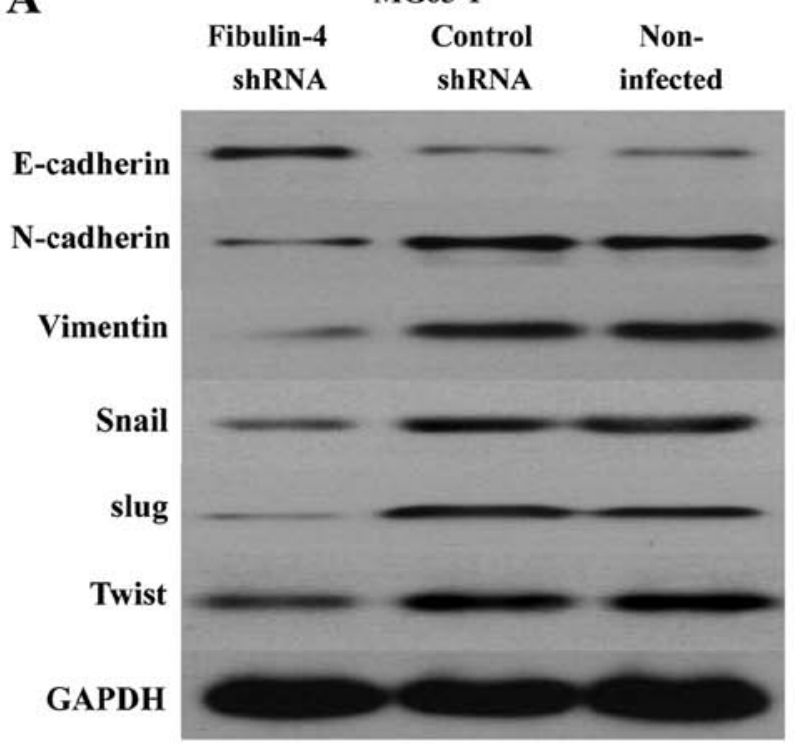

B

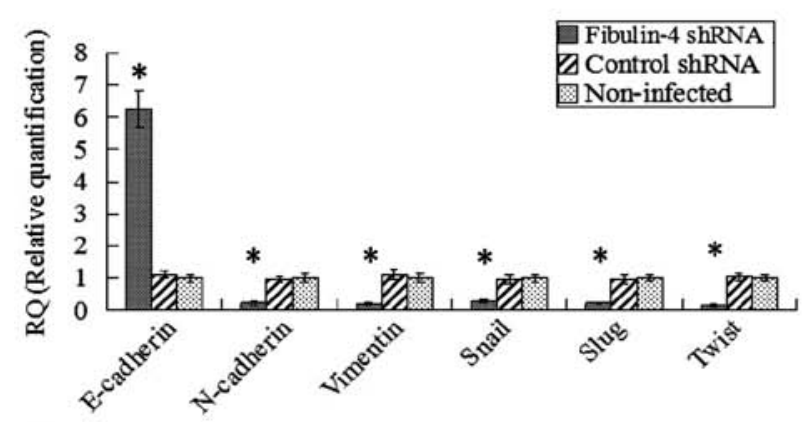

C

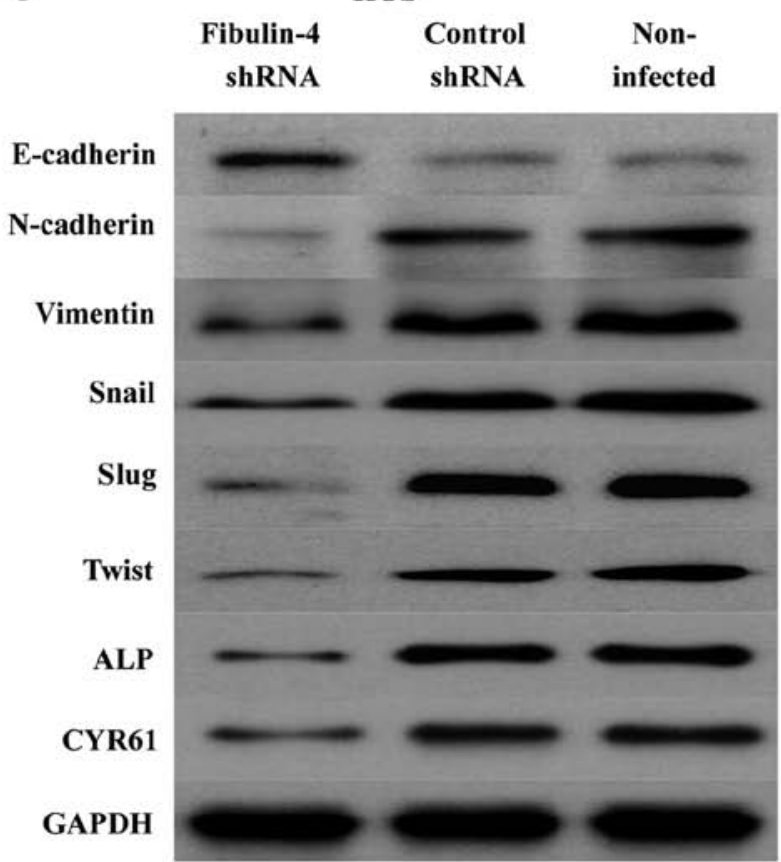

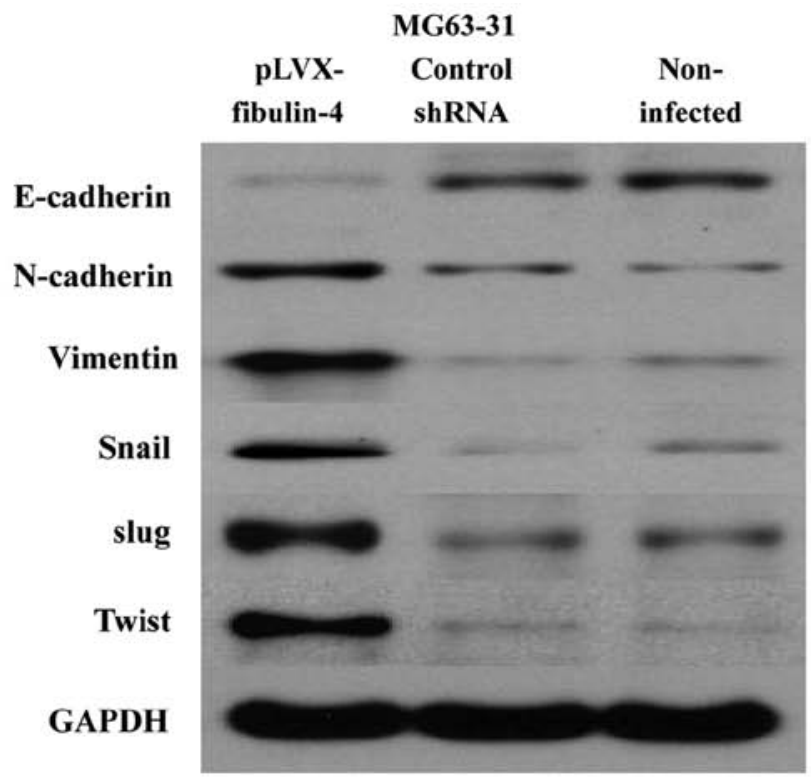

MG63-31

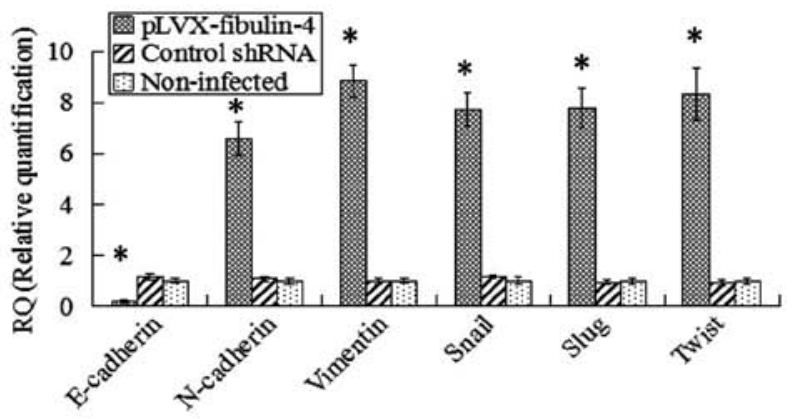

U-2OS

$\begin{array}{rlr}\text { pLVX- } & \text { Control } & \text { Non- } \\ \text { fibulin-4 } & \text { shRNA } & \text { infected }\end{array}$

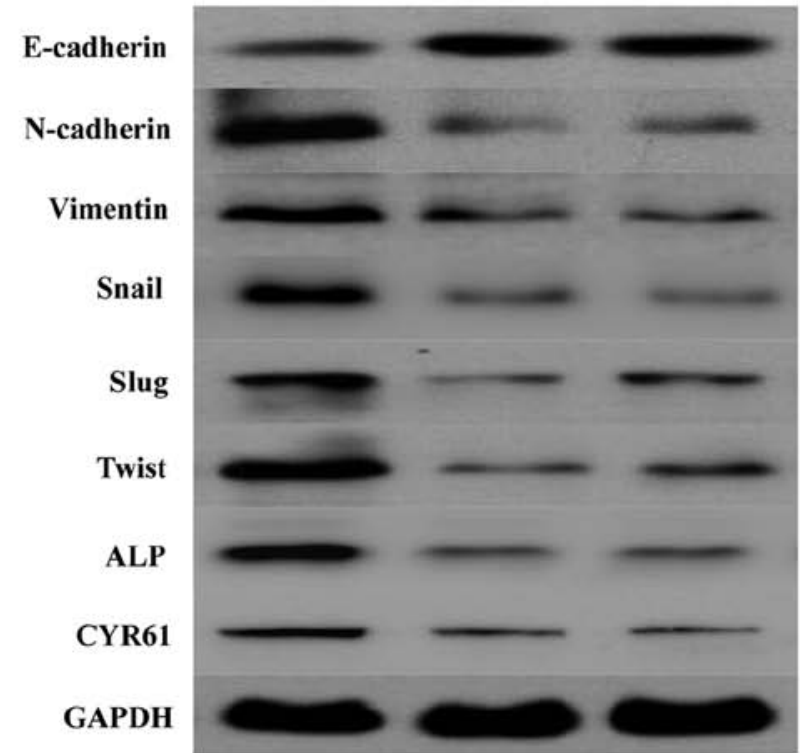

Figure 10. Effects of fibulin-4 knockdown and overexpression on EMT genes, CYR61 and ALP, which were correlated to tumor progression. After lentivirus transfections, EMT markers, including E-cadherin, N-cadherin, vimentin, Snail, Slug and Twist were measured by (A) western blotting and (B) real-time q-RT-PCR in the lentivirus transfection systems of the differently invasive osteosarcoma cell subclones. (C) EMT markers (E-cadherin, N-cadherin, vimentin, Snail, Slug and Twist), CYR61 and ALP were detected by western blotting in fibulin-4 shRNA-infected HOS cells and pLVX-fibulin-4-infected U-2OS cells. Fibulin-4 knockdown significantly increased the expression of E-cadherin, and decreased the expression of N-cadherin, vimentin, Snail, Slug and Twist; whereas fibulin-4 upregulation decreased the expression of E-cadherin, and increased the expression of N-cadherin, vimentin, Snail, Slug and Twist. CYR61 and ALP were downregulated following fibulin-4 shRNA infection, in association with the knockdown of fibulin-4 expression, moreover, with fibulin-4 upregulation, CYR61 and ALP were increased following pLVX-fibulin- 4 infection. ${ }^{*} \mathrm{P}<0.05$. 
A

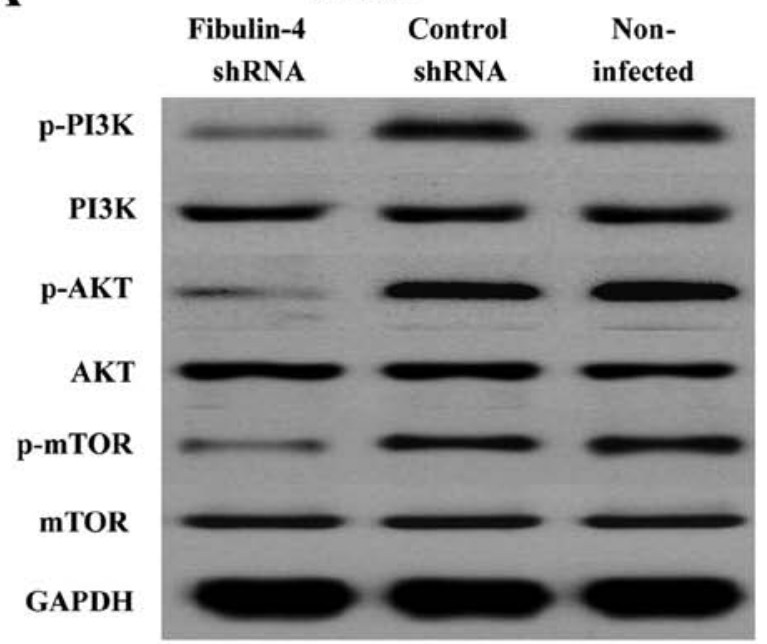

B
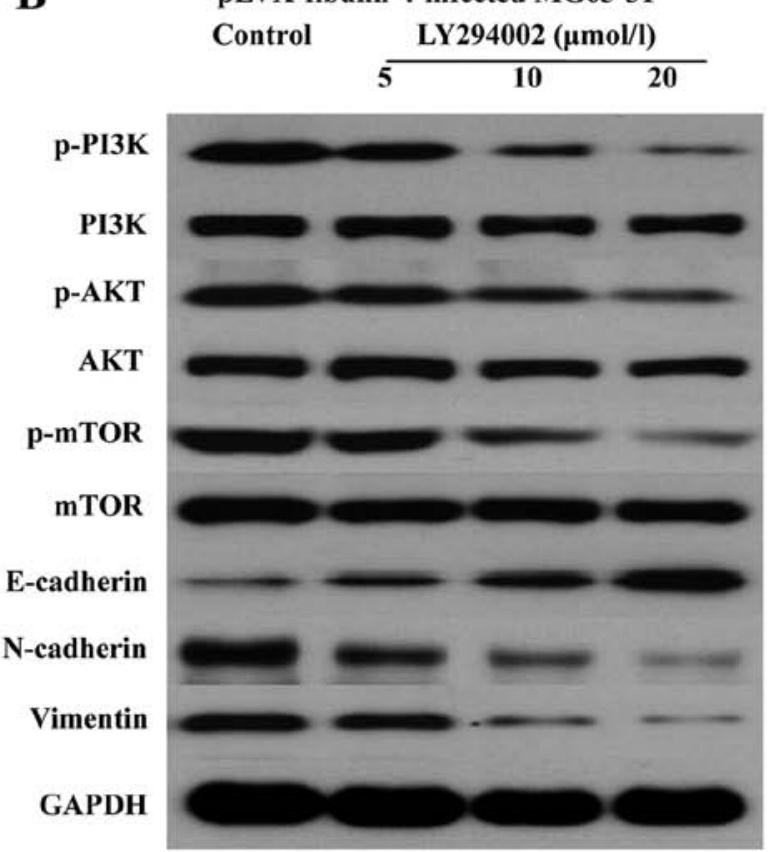

C

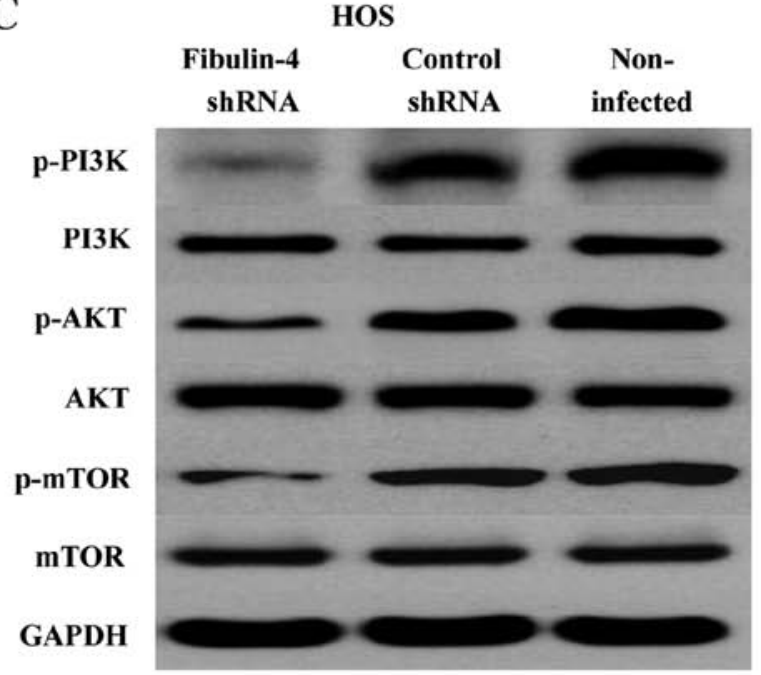

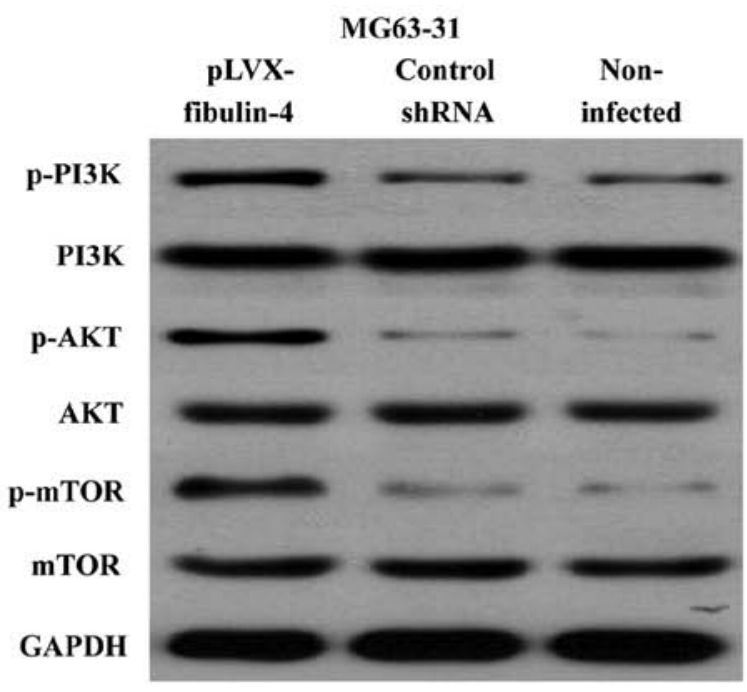

pLVX-fibulin-4-infected U-2OS
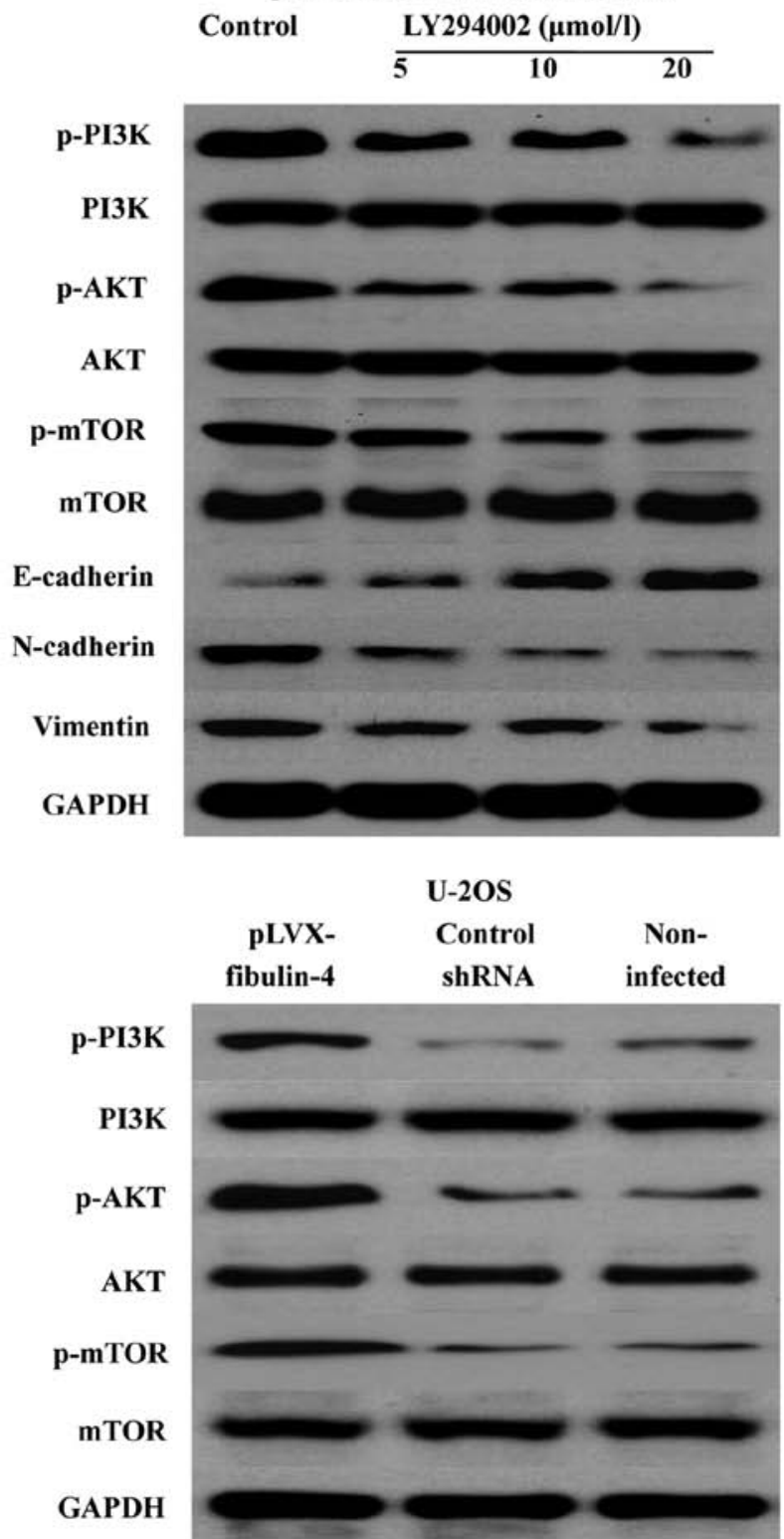

Figure 11. Effects of fibulin-4 on the PI3K/AKT signaling pathway. (A) In fibulin-4 shRNA-infected MG63-1 cells, fibulin-4 knockdown reduced the PI3K, AKT, and mTOR phosphorylation levels; in contrast, in pLVX-fibulin-4-infected MG63-31 cells, fibulin-4 upregulation increased their phosphorylation levels. (B) The PI3K/AKT signaling pathway inhibitor LY294002 significantly inhibited the PI3K/AKT pathway, and EMT, both of which were activated by fibulin-4 upregulation. (C) In fibulin-4 shRNA-infected HOS cells, fibulin-4 knockdown could reduce the PI3K, AKT, and mTOR phosphorylation levels; in contrast, in pLVX-fibulin-4-infected U-2OS cells, fibulin-4 upregulation could increase their phosphorylation levels. 
using real-time q-RT-PCR and western blotting, fibulin-4 knockdown significantly inhibited the process of EMT, accompanied with increased E-cadherin expression and decreased expression of N-cadherin, vimentin, Snail, Slug, and Twist; in contrast, fibulin-4 upregulation could induce EMT, with decreased E-cadherin expression, and increased expression of N-cadherin, vimentin, Snail, Slug, and Twist. The expression levels of CYR61 and ALP were also detected by western blotting in fibulin-4 shRNA-infected and pLVXfibulin-4-infected cells. CYR61 and ALP were downregulated following fibulin-4 shRNA infection, in association with the knockdown of fibulin-4 expression, at the same time, with fibulin-4 upregulation, CYR61 and ALP were increased following pLVX-fibulin-4 infection. In conclusion, fibulin-4 could promote the progression of human osteosarcomas.

Effects of fibulin-4 on the PI3K/AKT signaling pathway. PI3K-AKT-mTOR is one of the major signaling pathways identified as important in cancer. We determined the expression of the main signaling molecules PI3K, AKT, and mTOR, and the changes in their phosphorylation levels. The results revealed that fibulin- 4 knockdown was able to reduce the PI3K, AKT, and mTOR phosphorylation levels; in contrast, fibulin-4 upregulation could increase their phosphorylation levels. Using the PI3K/AKT signaling pathway inhibitor LY294002 $(5,10$, and $20 \mu \mathrm{mol} / 1)$, we treated the pLVXfibulin-4-infected cells for $48 \mathrm{~h}$. We found that the inhibitor could significantly inhibit the PI3K/AKT pathway, and EMT, both of which were activated by fibulin-4 upregulation. Concentration-dependent increases were observed with increasing doses (Fig. 11). In summary, fibulin-4 could induce EMT to promote osteosarcoma invasion and metastasis by the PI3K/AKT signaling pathway.

\section{Discussion}

In our study, we observed that high fibulin-4 expression was associated with poor prognosis of human osteosarcoma, and that fibulin- 4 was overexpressed in the highly invasive osteosarcoma cell line and subclone. Fibulin-4 promoted osteosarcoma cell invasion and metastasis by inducing EMT via the $\mathrm{PI} 3 \mathrm{~K} / \mathrm{AKT} / \mathrm{mTOR}$ pathway.

From the protein and mRNA levels, we found that fibulin-4 expression in osteosarcoma tissues and cell lines was much higher than that in normal tissues and cell lines, especially in the highly metastatic osteosarcoma cell line and subclone. Moreover, high fibulin-4 expression was detected in low differentiation and positive lymph node status. Similar results have been reported by Li and Wang (32), wherein compared to normal specimens and cell lines, upregulated fibulin-4 expression was found in osteosarcoma clinical specimens and cell lines. In colon cancer, fibulin-4 expression was enhanced, and it seemed to verify the hypothesis of fibulin-4 as an oncogene (33). After further studies, fibulin-4 was found to be a promising serum biomarker for the early detection of colorectal cancer (34).

In a study of gynecologic tumors, fibulin-4 overexpression was associated with tumor progression and poor prognosis in patients with cervical carcinoma and ovarian cancer $(29,35)$. In gliomas, fibulin-4 expression was significantly increased in the tumor tissues compared to the non-tumorous brain tissue (36). However, fibulin-4 expression was found to be decreased in prostate cancer (11). Therefore, we suspect that like some members of the fibulin family, the role of fibulin-4 in tumor development may exhibit tissue specificity, wherein different tumor microenvironments determine different gene functions (37). CYR61 is a secreted, extracellular matrix (ECM)-associated signaling protein of the CCN family. CYR61 is capable of regulating a broad range of cellular activities, including cell adhesion, migration, proliferation, differentiation, apoptosis, and senescence $(38,39)$. A previous study showed that Cyr61 expression is related to osteosarcoma progression. In addition, Cyr61 could promote cell migration and metastasis in osteosarcoma $(40,41)$. In the present study, by IHC, the expression levels of CYR61 and ALP in osteosarcoma tissues were significantly high. According to the Pearson's product-moment correlation coefficient, the expression of CYR61 and fibulin-4 exhibited strong positive correlations. In conclusion, we considered that fibulin-4 acted as a facilitator for osteosarcoma to promote tumor growth and dissemination.

Epithelial-mesenchymal transition (EMT) is a highly conserved cellular program by which epithelial cells lose cell polarity and cell-cell adhesion, and gain migratory and invasive properties thus converting to motile mesenchymal cells. This important process was initially recognized as a feature of embryogenesis and promotes carcinoma invasion and metastasis (42). Our results revealed that fibulin-4 knockdown suppressed the process of EMT, with upregulated expression of the epithelial marker E-cadherin, and decreased expression of the mesenchymal markers $\mathrm{N}$-cadherin and vimentin; whereas fibulin-4 upregulation promoted EMT, accompanied by decreased E-cadherin expression, and increased expression of N-cadherin and vimentin. The transcription factors Snail (Snail-1), Slug (Snail-2), and Twist which are involved in the process of EMT were downregulated in fibulin- 4 shRNA infected cells, and upregulated in pLVX-fibulin-4-infected cells. These data simultaneously indicate that fibulin- 4 could promote the process of EMT by affecting transcription factors, and thereby induce the invasion and metastasis of osteosarcoma cells. The role of fibulin-4 in the process of EMT has not yet been reported in other cancers.

The PI3K/AKT/mTOR pathway is an intracellular signaling pathway directly related to cellular quiescence, proliferation, cancer, and longevity. In many cancers, this pathway is overactive, thus reducing apoptosis, allowing proliferation, and promoting invasion and metastasis $(43,44)$. In our study, overexpression levels of fibulin-4 could upregulate the phospho-PI3K, phospho-AKT, and phospho-mTOR activities and promote the process of EMT, whereas knockdown of its expression could significantly downregulate phospho-PI3K, phospho-AKT, and phospho-mTOR activities and inhibit EMT. Furthermore, we found that the PI3K/AKT signaling pathway inhibitor LY294002 significantly inhibited the PI3K/AKT pathway and EMT, both of which were activated by fibulin-4 upregulation. This study demonstrated for the first time that fibulin-4 could promote EMT through the PI3K/AKT/mTOR signaling pathway. As the research on fibulin-4 is still in its infancy, we could not find similar reports in other cancers. However, EFEMP1, which shares 
high homology with fibulin-4 (45), affects the development of a variety of tumors through the PI3/AKT pathway $(19,46,47)$.

In conclusion, high fibulin-4 expression was associated with poor prognosis in human osteosarcoma and the malignant phenotype of osteosarcoma cells. Fibulin-4 has the ability to promote proliferation, invasion, and metastasis of osteosarcoma cells by inducing EMT through the PI3K/AKT/mTOR signaling pathway. We believe that further research on fibulin-4 can contribute to more effective treatment for inhibiting invasion and metastasis of osteosarcoma.

\section{Acknowledgements}

The study was supported by the fund of Suzhou Science and Technology Project (XJ201536).

\section{References}

1. Geller DS and Gorlick R: Osteosarcoma: A review of diagnosis, management, and treatment strategies. Clin Adv Hematol Oncol 8: 705-718, 2010.

2. Messerschmitt PJ, Rettew AN, Brookover RE, Garcia RM, Getty PJ and Greenfield EM: Specific tyrosine kinase inhibitors regulate human osteosarcoma cells in vitro. Clin Orthop Relat Res 466: 2168-2175, 2008.

3. de Vega S, Iwamoto T and Yamada Y: Fibulins: Multiple roles in matrix structures and tissue functions. Cell Mol Life Sci 66 1890-1902, 2009.

4. Kobayashi N, Kostka G, Garbe JH, Keene DR, Bächinger HP, Hanisch FG, Markova D, Tsuda T, Timpl R, Chu ML, et al: A comparative analysis of the fibulin protein family. Biochemical characterization, binding interactions, and tissue localization. $\mathrm{J}$ Biol Chem 282: 11805-11816, 2007.

5. Papke CL and Yanagisawa H: Fibulin-4 and fibulin-5 in elastogenesis and beyond: Insights from mouse and human studies. Matrix Biol 37: 142-149, 2014.

6. Obaya AJ, Rua S, Moncada-Pazos A and Cal S: The dual role of fibulins in tumorigenesis. Cancer Lett 325: 132-138, 2012.

7. Moll F, Katsaros D, Lazennec G, Hellio N, Roger P, Giacalone PL, Chalbos D, Maudelonde T, Rochefort $\mathrm{H}$ and Pujol P: Estrogen induction and overexpression of fibulin-1C mRNA in ovarian cancer cells. Oncogene 21: 1097-1107, 2002.

8. Greene LM, Twal WO, Duffy MJ, McDermott EW, Hill AD, O'Higgins NJ, McCann AH, Dervan PA, Argraves WS and Gallagher WM: Elevated expression and altered processing of fibulin-1 protein in human breast cancer. Br J Cancer 88 871-878, 2003

9. Kanda M, Nomoto S, Okamura Y, Hayashi M, Hishida M, Fujii T, Nishikawa Y, Sugimoto H, Takeda S and Nakao A: Promoter hypermethylation of fibulin 1 gene is associated with tumor progression in hepatocellular carcinoma. Mol Carcinog 50: 571-579, 2011.

10. Cheng YY, Jin H, Liu X, Siu JM, Wong YP, Ng EK, Yu J, Leung WK, Sung JJ and Chan FK: Fibulin 1 is downregulated through promoter hypermethylation in gastric cancer. Br J Cancer 99: 2083-2087, 2008.

11. Wlazlinski A, Engers R, Hoffmann MJ, Hader C, Jung V, Müller M and Schulz WA: Downregulation of several fibulin genes in prostate cancer. Prostate 67: 1770-1780, 2007.

12. Xie L, Palmsten K, MacDonald B, Kieran MW, Potenta S, Vong $S$ and Kalluri R: Basement membrane derived fibulin-1 and fibulin-5 function as angiogenesis inhibitors and suppress tumor growth. Exp Biol Med (Maywood) 233: 155-162, 2008.

13. Law EW, Cheung AK, Kashuba VI, Pavlova TV, Zabarovsky ER, Lung HL, Cheng Y, Chua D, Lai-Wan Kwong D, Tsao SW, et al: Anti-angiogenic and tumor-suppressive roles of candidate tumor-suppressor gene, Fibulin-2, in nasopharyngeal carcinoma. Oncogene 31: 728-738, 2012

14. Yi CH, Smith DJ, West WW and Hollingsworth MA: Loss of fibulin-2 expression is associated with breast cancer progression. Am J Pathol 170: 1535-1545, 2007.
15. Seeliger H, Camaj P, Ischenko I, Kleespies A, De Toni EN, Thieme SE, Blum H, Assmann G, Jauch KW and Bruns CJ: EFEMP1 expression promotes in vivo tumor growth in human pancreatic adenocarcinoma. Mol Cancer Res 7: 189-198, 2009.

16. Song EL, Hou YP, Yu SP, Chen SG, Huang JT, Luo T, Kong LP, $\mathrm{Xu} \mathbf{J}$ and Wang HQ: EFEMP1 expression promotes angiogenesis and accelerates the growth of cervical cancer in vivo. Gynecol Oncol 121: 174-180, 2011.

17. En-lin S, Sheng-guo C and Hua-qiao W: The expression of EFEMP1 in cervical carcinoma and its relationship with prognosis. Gynecol Oncol 117: 417-422, 2010.

18. Hu B, Thirtamara-Rajamani KK, Sim H and Viapiano MS: Fibulin-3 is uniquely upregulated in malignant gliomas and promotes tumor cell motility and invasion. Mol Cancer Res 7: 1756-1770, 2009.

19. Hwang CF, Chien CY, Huang SC, Yin YF, Huang CC, Fang FM, Tsai HT, Su LJ and Chen CH: Fibulin-3 is associated with tumour progression and a poor prognosis in nasopharyngeal carcinomas and inhibits cell migration and invasion via suppressed AKT activity. J Pathol 222: 367-379, 2010.

20. Sadr-Nabavi A, Ramser J, Volkmann J, Naehrig J, Wiesmann F, Betz B, Hellebrand H, Engert S, Seitz S, Kreutzfeld R, et al: Decreased expression of angiogenesis antagonist EFEMP1 in sporadic breast cancer is caused by aberrant promoter methylation and points to an impact of EFEMP1 as molecular biomarker. Int J Cancer 124: 1727-1735, 2009.

21. Hu Y, Pioli PD, Siegel E, Zhang Q, Nelson J, Chaturbedi A, Mathews MS, Ro DI, Alkafeef S, Hsu N, et al: EFEMP1 suppresses malignant glioma growth and exerts its action within the tumor extracellular compartment. Mol Cancer 10: 123, 2011.

22. Schluterman MK, Chapman SL, Korpanty G, Ozumi K, Fukai T, Yanagisawa $\mathrm{H}$ and Brekken RA: Loss of fibulin-5 binding to betal integrins inhibits tumor growth by increasing the level of ROS. Dis Model Mech 3: 333-342, 2010.

23. Hu Z, Ai Q, Xu H, Ma X, Li HZ, Shi TP, Wang C, Gong DJ and Zhang X: Fibulin-5 is down-regulated in urothelial carcinoma of bladder and inhibits growth and invasion of human bladder cancer cell line 5637. Urol Oncol 29: 430-435, 2011.

24. Yue W, Sun Q, Landreneau R, Wu C, Siegfried JM, Yu J and Zhang L: Fibulin-5 suppresses lung cancer invasion by inhibiting matrix metalloproteinase-7 expression. Cancer Res 69: 6339-6346, 2009.

25. Albig AR and Schiemann WP: Fibulin-5 function during tumorigenesis. Future Oncol 1: 23-35, 2005.

26. Omasu F, Nakano Y and Ichiki T: Measurement of the electrophoretic mobility of sheep erythrocytes using microcapillary chips. Electrophoresis 26: 1163-1167, 2005.

27. Chen J, Zhang J, Zhao Y, Li J and Fu M: Integrin beta3 downregulates invasive features of ovarian cancer cells in SKOV3 cell subclones. J Cancer Res Clin Oncol 135: 909-917, 2009.

28. Chang XZ, Wang ZM, Yu JM, Tian FG, Jin W, Zhang Y, Yu J, $\mathrm{Li} \mathrm{LF}$, Liu XF, Li ZW, et al: Isolation of a human gallbladder cancer cell clone with high invasive phenotype in vitro and metastatic potential in orthotopic model and inhibition of its invasiveness by heparanase antisense oligodeoxynucleotides. Clin Exp Metastasis 24: 25-38, 2007.

29. Chen J, Liu Z, Fang S, Fang R, Liu X, Zhao Y, Li X, Huang L and Zhang J: Fibulin-4 is associated with tumor progression and a poor prognosis in ovarian carcinomas. BMC Cancer 15: 91, 2015.

30. Yang X, Li D, Cheng S, Fan K, Sheng L, Zhang J, Feng B and $\mathrm{Xu} Z$ : The correlation of bone morphogenetic protein 2 with poor prognosis in glioma patients. Tumour Biol 35: 11091-11095, 2014.

31. Albini A, Iwamoto Y, Kleinman HK, Martin GR, Aaronson SA, Kozlowski JM and McEwan RN: A rapid in vitro assay for quantitating the invasive potential of tumor cells. Cancer Res 47: 3239-3245, 1987

32. Li R and Wang L: Fibulin-4 is a novel Wnt//-catenin pathway activator in human osteosarcoma. Biochem Biophys Res Commun 474: 730-735, 2016.

33. Gallagher WM, Greene LM, Ryan MP, Sierra V, Berger A, Laurent-Puig P and Conseiller E: Human fibulin-4: Analysis of its biosynthetic processing and mRNA expression in normal and tumour tissues. FEBS Lett 489: 59-66, 2001.

34. Yao L, Lao W, Zhang Y, Tang X, Hu X, He C, Hu X and Xu LX: Identification of EFEMP2 as a serum biomarker for the early detection of colorectal cancer with lectin affinity capture assisted secretome analysis of cultured fresh tissues. J Proteome Res 11: 3281-3294, 2012. 
35. Chen J,Zhang J, Liu X, Fang R, Zhao Y and Ma D: Overexpression of fibulin-4 is associated with tumor progression and poor prognosis in patients with cervical carcinoma. Oncol Rep 31: 2601-2610, 2014.

36. Wang L, Chen Q, Chen Z, Tian D, Xu H, Cai Q, Liu B and Deng G: EFEMP2 is upregulated in gliomas and promotes glioma cell proliferation and invasion. Int J Clin Exp Pathol 8: 10385-10393, 2015.

37. Chen L, Sun B, Zhang S, Zhao X, He Y, Zhao S, Lin T and Li X: Influence of microenvironments on microcirculation patterns and tumor invasion-related protein expression in melanoma. Oncol Rep 21: 917-923, 2009.

38. Lau LF: CCN1/CYR61: The very model of a modern matricellular protein. Cell Mol Life Sci 68: 3149-3163, 2011.

39. Lau LF: Cell surface receptors for CCN proteins. J Cell Commun Signal 10: 121-127, 2016.

40. Hou CH, Lin FL, Hou SM and Liu JF: Cyr61 promotes epithelialmesenchymal transition and tumor metastasis of osteosarcoma by Raf-1/MEK/ERK/Elk-1/TWIST-1 signaling pathway. Mol Cancer 13: 236, 2014

41. Habel N, Vilalta M, Bawa O, Opolon P, Blanco J and Fromigué O: Cyr61 silencing reduces vascularization and dissemination of osteosarcoma tumors. Oncogene 34: 3207-3213, 2015.
42. Singh A and Settleman J: EMT, cancer stem cells and drug resistance: An emerging axis of evil in the war on cancer. Oncogene 29: 4741-4751, 2010.

43. Li X, Wu C, Chen N, Gu H, Yen A, Cao L, Wang E and Wang L: $\mathrm{PI} 3 \mathrm{~K} / \mathrm{Akt} / \mathrm{mTOR}$ signaling pathway and targeted therapy for glioblastoma. Oncotarget 7: 33440-33450, 2016.

44. Gao Y, Yuan CY and Yuan W: Will targeting PI3K/Akt/mTOR signaling work in hematopoietic malignancies? Stem Cell Investig 3: 31, 2016.

45. Zhang Y and Marmorstein LY: Focus on molecules: Fibulin-3 (EFEMP1). Exp Eye Res 90: 374-375, 2010.

46. Kim IG, Kim SY, Choi SI, Lee JH, Kim KC and Cho EW: Fibulin-3-mediated inhibition of epithelial-to-mesenchymal transition and self-renewal of $\mathrm{ALDH}^{+}$lung cancer stem cells through IGF1R signaling. Oncogene 33: 3908-3917, 2014.

47. Camaj P, Seeliger H, Ischenko I, Krebs S, Blum H, De Toni EN, Faktorova D, Jauch KW and Bruns CJ: EFEMP1 binds the EGF receptor and activates MAPK and Akt pathways in pancreatic carcinoma cells. Biol Chem 390: 1293-1302, 2009. 